\title{
Gaussian operator bases for correlated fermions
}

\author{
J F Corney and P D Drummond \\ ARC Centre of Excellence for Quantum-Atom Optics, University of Queensland, Brisbane 4072, \\ Queensland, Australia
}

Received 3 August 2005

Published 14 December 2005

Online at stacks.iop.org/JPhysA/39/269

\begin{abstract}
We formulate a general multi-mode Gaussian operator basis for fermions, to enable a positive phase-space representation of correlated Fermi states. The Gaussian basis extends existing bosonic phase-space methods to Fermi systems and thus allows first-principles dynamical or equilibrium calculations in quantum many-body Fermi systems. We prove the completeness of the basis and derive differential forms for products with one- and two-body operators. Because the basis satisfies fermionic superselection rules, the resulting phase space involves only $c$-numbers, without requiring anticommuting Grassmann variables. Furthermore, because of the overcompleteness of the basis, the phase-space distribution can always be chosen positive. This has important consequences for the sign problem in fermion physics.
\end{abstract}

PACS numbers: $\quad$ 03.65.Ca, 42.50.Lc, 03.75.Ss, 71.10.Fd

\section{Introduction}

In this paper, we address the issue of how to represent highly correlated fermionic states, for the purposes of efficient calculations in fermionic many-body physics. To this end, we introduce a normally ordered Gaussian operator basis for fermionic density operators, in order to extend earlier phase-space techniques used to represent atomic transitions $[1,2]$. The analogous phase-space representations for bosons were first introduced over a classical phase space [3-6], which were subsequently extended to a non-classical phase space $[7,8]$ and then to a complete Gaussian basis [9].

We show that, just as with bosons, the Gaussian basis enables a representation of arbitrary fermionic density operators as a positive distribution over a generalized phase space. We investigate both a number-conserving basis set, which includes the usual thermal states used to describe Fermi gases, and a non-number-conserving basis set, which also includes the Bardeen-Cooper-Schreiffer (BCS) states found in superconductivity theory [10]. The latter comprises the most general Gaussian basis. This approach has the advantage that the phasespace variables correspond to covariances or Green's functions, described by coordinate pairs. 
Unlike many previous antisymmetric wavefunction or path-integral approaches, distributions over the covariances can be chosen as probabilities, which therefore allow the use of random sampling without the usual fermionic sign problem.

In this paper, we concentrate on the foundational issues of the Gaussian representation method, proving three central results:

- Gaussian operators form a complete basis for any physical density matrix,

- the resulting distribution can always be chosen positive,

- all two-body operators map to a second-order differential form.

From these results, it follows that positive-definite Fokker-Planck equations [11] exist for many-body fermionic systems. Such Fokker-Planck equations enable first-principles stochastic numerical simulation methods, either in real time or at finite temperature. These have already been applied to the important and fundamental case of the finite temperature Hubbard model $[12,13]$, which has well-known sign problems when treated using path integrals. In this case, the Gaussian method does not suffer from the usual sign problems, because the distributions and weights can always be chosen to be positive. Stochastic equations can also be used to obtain novel types of perturbation theory using stochastic diagram techniques [14]. The detailed formulation of the resultant phase-space simulation methods will be given elsewhere [15].

This approach has some similarities to auxiliary field methods [16] used to treat fermionic path integrals. However, such path integrals are not generally guaranteed to correspond to probabilities. The advantage of the present approach is that it is applied to directly expand the fermionic density operators. This, in turn, allows a greater physical understanding and fewer restrictions in the resulting applications. Because phasespace methods embody a different approach to quantum mechanics than path integrals, a number of important theorems and identities need to be established. These new results, pertaining to completeness, positivity and differential identities, are given in the present paper.

To begin with, we establish in section 2 the definition of a Gaussian operator in unnormalized form. We follow this in section 3 with elementary examples of one- and twomode Gaussians in order to illustrate the basic structure and the physics underlying it. The two-mode examples include the density operators for thermal and squeezed/BCS fermionic states. The squeezed fermionic states correspond physically to systems with broken symmetry in condensed matter. In section 4, we introduce some notation that is convenient for discussing general Gaussian operators in a multi-mode system.

The main part of the paper (section 5) introduces the most general class of multi-mode normalized Gaussian operators and discusses those of its properties that are relevant to its use as an operator basis set. This section gives the proofs of completeness and positivity properties, by means of expansions in number-state projectors. Depending on the phase properties of the density matrix, expansions may require either phase-insensitive (normal) Green's functions or phase-sensitive (anomalous) Green's functions. We also derive a number of differential identities, which are proved in appendix B by use of Fermi coherent states [17] (appendix A). These are relevant to the derivation of Fokker-Planck and stochastic equations, which are explained in detail in [15].

\section{Definitions}

To define Gaussian operators for a given fermionic system, we first decompose it into a set of $M$ orthogonal single-particle modes or orbitals. With each of these modes, we associate 
creation and annihilation operators $\widehat{b}_{j}^{\dagger}$ and $\widehat{b}_{j}$, with anticommutation relations

$$
\left[\widehat{b}_{j}, \widehat{b}_{k}^{\dagger}\right]_{+}=\delta_{j k}, \quad\left[\widehat{b}_{j}, \widehat{b}_{k}\right]_{+}=0,
$$

where $j, k=1, \ldots, M$. Thus, $\widehat{\boldsymbol{b}}$ is a column vector of the $M$ annihilation operators and $\widehat{\boldsymbol{b}}^{\dagger}$ is a row vector of the corresponding creation operators.

We define a Gaussian operator to be a normally ordered, Gaussian form of annihilation and creation operators. Like a complex-number Gaussian, the operator Gaussian is an exponential of a quadratic form, with the exponential defined by its series representation. We follow the standard convention that fermionic normal ordering includes a sign change for every operator exchange, so that $: \widehat{b}_{k} \widehat{b}_{j}^{\dagger}:=-\widehat{b}_{j}^{\dagger} \widehat{b}_{k}$. Normal ordering is utilized here because it allows us to most directly use Fermi coherent-state methods [17, 18] involving Grassmann algebra $[19,20]$, which are described in greater detail in the appendices. We note that from particle-hole symmetry, it is also possible to obtain an entirely analogous antinormally ordered representation, which simply exchanges the roles of particles and holes in the formalism.

The most general Gaussian form is a cumbersome object to manipulate, unless products of odd numbers of operators are excluded. Fortunately, restricting the set of Gaussians to those containing only even products can be physically justified on the basis of superselection rules for fermions. Because it is constructed from pairs of operators, this type of Gaussian operator contains no Grassmann variables, in contrast to the Fermi representations in [18] and [21].

In order to relate these quadratic expressions to the usual Dirac state-vector notation in the examples that follow, we define number states as

$$
\begin{aligned}
|\vec{n}\rangle & =\left|n_{1}\right\rangle \otimes\left|n_{2}\right\rangle \cdots \otimes\left|n_{M}\right\rangle \\
& =\left(\widehat{b}_{1}^{\dagger}\right)^{n_{1}}\left(\widehat{b}_{2}^{\dagger}\right)^{n_{2}} \cdots\left(\widehat{b}_{M}^{\dagger}\right)^{n_{M}}|0\rangle_{M},
\end{aligned}
$$

where $\vec{n}=\left(n_{1}, n_{2}, \ldots, n_{M}\right)$ is a vector of integer occupations and where $|0\rangle_{M}$ is the $M$-mode fermion vacuum state.

We also recall some elementary identities which relate quadratic forms in fermion operators to state projection operators. For individual operators, one has the well-known identities:

$$
\widehat{b}_{j}=|0\rangle_{j}\left\langle\left. 1\right|_{j}, \quad \widehat{b}_{j}^{\dagger}=\mid 1\right\rangle_{j}\left\langle\left. 0\right|_{j} .\right.
$$

Hence, the elementary identities for quadratic products are as follows, for $i<j$,

$$
\begin{aligned}
& \widehat{b}_{i}^{\dagger} \widehat{b}_{i}=|1\rangle_{i}\left\langle\left. 1\right|_{i} \prod_{k \neq i} \hat{1}_{k}\right. \\
& \widehat{b}_{i} \widehat{b}_{i}^{\dagger}=|0\rangle_{i}\left\langle\left. 0\right|_{i} \prod_{k \neq i} \hat{1}_{k}\right. \\
& \widehat{b}_{i}^{\dagger} \widehat{b}_{j}=\left|1_{i}, 0_{j}\right\rangle\left\langle 0_{i}, 1_{j}\right| \prod_{k \neq i, j} \hat{1}_{k} \\
& \widehat{b}_{j} \widehat{b}_{i}=\left|0_{i}, 0_{j}\right\rangle\left\langle 1_{i}, 1_{j}\right| \prod_{k \neq i, j} \hat{1}_{k},
\end{aligned}
$$

where $\hat{1}_{k}$ is the identity operator for the $k$ th mode.

Expanding quantum states in terms of an underlying basis set is a widespread procedure in quantum mechanics. However, using the general Gaussian operators in this capacity is nonstandard in several respects. First, one is expanding a quantum density operator over a basis set that includes mixed as well as pure quantum states. (By 'quantum state', we mean density operators rather than state vectors, even for pure states.) Second, the Gaussian 
operator basis states are not orthogonal. Third, we also allow the basis set to include operators which are not themselves density operators. These additional degrees of freedom prove useful in obtaining the requisite completeness properties. We now give the mathematical form of these basis operators, in unnormalized form. Normalized forms $\widehat{\Lambda}_{M}$ will be introduced in section 5 .

\subsection{Number-conserving Gaussians}

We first consider the special case obtained when the quadratic is of form $\widehat{b}_{i}^{\dagger} \widehat{b}_{j}$, so that it is number conserving. The most general, number-conserving Gaussian operator can be written as

$$
\begin{aligned}
\widehat{\Lambda}_{M}^{(u)}(\boldsymbol{\mu}) & =: \exp \left[-\widehat{\boldsymbol{b}}^{\dagger} \boldsymbol{\mu} \widehat{\boldsymbol{b}}\right]: \\
& =: \prod_{i, j=1}^{M} \exp \left[-\widehat{b}_{i}^{\dagger} \mu_{i j} \widehat{b}_{j}\right]: \\
& =: \prod_{i, j=1}^{M}\left[\hat{1}-\widehat{b}_{i}^{\dagger} \mu_{i j} \widehat{b}_{j}\right]:
\end{aligned}
$$

where $\boldsymbol{\mu}$ is a complex $M \times M$ matrix. The last result follows because normally ordered products in which the same operator appears more than once are zero, from the anticommutation relations in equation (2.1). For the special case that $\boldsymbol{\mu}$ is a Hermitian matrix, the Gaussian operator is just the density operator of a thermal state. If all the eigenvalues of $\boldsymbol{\mu}$ are also either 0 or 1 , then the Gaussian corresponds to a Slater determinant (i.e., a product of single-mode number states).

It should be noted here that we do not restrict the Gaussian operators of this type to be just thermal states. In general, we would wish to consider density matrices that are linear combinations of Gaussian operators. These can exist as Hermitian, positive-definite operators even when composed of Gaussians that have neither property.

\subsection{Non-number-conserving Gaussians}

If anomalous products of form $\widehat{b}_{i} \widehat{b}_{j}$ are included as well, then the most general non-numberconserving Gaussian is a type of squeezed state:

$$
\begin{aligned}
\widehat{\Lambda}_{M}^{(u)}\left(\boldsymbol{\mu}, \boldsymbol{\xi}, \boldsymbol{\xi}^{+}\right) & =: \exp \left[-\widehat{\boldsymbol{b}}^{\dagger} \boldsymbol{\mu} \widehat{\boldsymbol{b}}-\frac{1}{2}\left(\widehat{\boldsymbol{b}} \boldsymbol{\xi}^{+} \widehat{\boldsymbol{b}}+\widehat{\boldsymbol{b}}^{\dagger} \boldsymbol{\xi} \widehat{\boldsymbol{b}}^{\dagger}\right)\right]: \\
& =\prod_{i>j}\left[1-\xi_{i j} \hat{b}_{i}^{\dagger} \hat{b}_{j}^{\dagger}\right]: \prod_{i j}\left[1-\mu_{i j} \hat{b}_{i}^{\dagger} \hat{b}_{j}\right]: \prod_{i>j} \exp \left[1-\xi_{i j}^{+} \hat{b}_{i} \hat{b}_{j}\right] .
\end{aligned}
$$

Here, $\boldsymbol{\xi}, \boldsymbol{\xi}^{+}$are complex antisymmetric $M \times M$ matrices. For the special case that $\boldsymbol{\mu}$ is a Hermitian matrix and $\xi^{\dagger}=\xi^{+}$, then the Gaussian operator is just the density operator for a squeezed thermal state.

As before, we do not restrict the Gaussian operators to be just the squeezed thermal states, even though these represent a large and physically important class of fermionic density operators. By extending the definition to include all exponentials of quadratic forms, we can obtain a more useful set, which is a complete basis with a positive-definite representation for all density operators, as we show in section 5 . 


\section{One- and two-mode Gaussian operators}

In this section, we give examples of Gaussian operators in elementary one- and two-mode cases. These are sufficient to illustrate the basic identities and ideas. More general results will be given in section 5 .

\subsection{Single-mode Gaussian operators}

An unnormalized Gaussian operator for a single mode has only one possible form:

$$
\begin{aligned}
\widehat{\Lambda}_{1}^{(u)}(\mu) & =: \exp \left[-\mu \widehat{b}^{\dagger} \widehat{b}\right]: \\
& \equiv: \sum_{k=0}^{\infty} \frac{1}{k !}\left(-\mu \widehat{b}^{\dagger} \widehat{b}\right)^{k}:
\end{aligned}
$$

where the exponential is defined, as indicated, by its series representation. Just as in the general case of equation (2.5), the anticommutivity of the fermionic operators means that only the zeroth- and first-order terms in the expansion contribute to the single-mode Gaussian, giving the simple result

$$
\widehat{\Lambda}_{1}^{(u)}(\mu)=1-\mu \widehat{b}^{\dagger} \widehat{b} .
$$

The normalization of this Gaussian operator is

$$
\begin{aligned}
N_{1} & \equiv \operatorname{Tr} \widehat{\Lambda}_{1} \\
& =2-\mu .
\end{aligned}
$$

Excluding the point $\mu=2$, we define new parameters $n=(1-\mu) /(2-\mu)$ and $\tilde{n}=1-n$, which allow us to write the Gaussian in normalized form as

$$
\begin{aligned}
\widehat{\Lambda}_{1}(n) & =\tilde{n}: \exp \left[-(2-1 / \tilde{n}) \widehat{b}^{\dagger} \widehat{b}\right]: \\
& =\tilde{n} \widehat{b}^{\dagger}+n \widehat{b}^{\dagger} \widehat{b} \\
& =\tilde{n}|0\rangle\langle 0|+n| 1\rangle\langle 1| .
\end{aligned}
$$

If $n$ is real, then equation (3.4) shows directly that $\widehat{\Lambda}_{1}(n)$ is the density operator corresponding to a mixture of number states in the one-mode case. The expectation value of the particle number $\widehat{n} \equiv \widehat{b}^{\dagger} \widehat{b}$ is just the variable $n$. Conversely, $\tilde{n}$ gives the number of holes.

\subsubsection{Completeness and positivity. As two special cases, we obtain the number states}

$$
|0\rangle\left\langle 0\left|=\widehat{\Lambda}_{1}(0), \quad\right| 1\right\rangle\langle 1|=\widehat{\Lambda}_{1}(1),
$$

which implies that just these two single-mode Gaussians form a complete basis set for a number-conserving subset of Hilbert space. Because superselection rules prohibit superpositions of states differing by odd numbers of fermions, this is the most general case possible. Furthermore, we see from equation (3.5) that the most general single-mode density matrix can be expanded as a mixture of Gaussians with positive coefficients, since $0 \leqslant n \leqslant 1$ :

$$
\widehat{\rho}=\tilde{n} \widehat{\Lambda}_{1}(0)+n \widehat{\Lambda}_{1}(1)
$$

Additionally in the one-mode case, all physical density operators are also Gaussian operators $\widehat{\rho}=\widehat{\Lambda}_{1}(n)$, which is another proof of positivity and completeness.

It is clear from these examples that the Gaussian operators are overcomplete: a physical density matrix may be represented by more than one positive distribution over the Gaussians. The most general single-mode Gaussian operator, with $n$ complex, provides an even larger overcomplete basis for physical density matrices, even though such Gaussians are not always 
physical density matrices themselves. For example, any uniform-phase distribution over Gaussian states gives the zero-particle state:

$$
|0\rangle\langle 0|=\int \mathrm{d} \phi \widehat{\Lambda}_{1}\left(r \mathrm{e}^{\mathrm{i} \phi}\right) .
$$

From the usual hole-particle symmetry of fermion states, the single-particle state is similarly obtained from

$$
|1\rangle\langle 1|=\int \mathrm{d} \phi \widehat{\Lambda}_{1}\left(1-r \mathrm{e}^{\mathrm{i} \phi}\right)
$$

\subsection{Two-mode number-conserving Gaussian operators}

A straightforward extension of the generalized thermal Gaussian form to two modes gives

$$
\begin{aligned}
\widehat{\Lambda}_{2}^{(u)}(\boldsymbol{\mu}) & =: \exp \left[-\sum_{i, j=1}^{2} \mu_{i j} \widehat{b}_{i}^{\dagger} \widehat{b}_{j}\right]: \\
& =1-\sum_{i, j=1}^{2} \mu_{i j} \widehat{b}_{i}^{\dagger} \widehat{b}_{j}+\operatorname{det}[\boldsymbol{\mu}] \widehat{b}_{1}^{\dagger} \widehat{b}_{2}^{\dagger} \widehat{b}_{2} \widehat{b}_{1},
\end{aligned}
$$

where the $\mu_{i j}$ are the elements of the $2 \times 2$ matrix $\mu$. The last step follows by explicitly expanding the general result in equation (2.5), while taking account of the sign changes during normal ordering. Again, the series expansion contains all possible normally ordered nonzero products of the $\widehat{b}_{i}^{\dagger} \hat{b}_{j}$ pairs. In terms of two-mode number-state projectors, the Gaussian operator is

$$
\begin{aligned}
\widehat{\Lambda}_{2}^{(u)}(\boldsymbol{\mu})=|00\rangle & \left\langle 00\left|+\left(1-\mu_{11}\right)\right| 10\right\rangle\left\langle 10\left|+\left(1-\mu_{22}\right)\right| 01\right\rangle\langle 01| \\
& +\left(1-\mu_{11}-\mu_{22}+\operatorname{det}[\boldsymbol{\mu}]\right)|11\rangle\left\langle 11\left|-\mu_{12}\right| 10\right\rangle\left\langle 01\left|-\mu_{21}\right| 01\right\rangle\langle 10| .
\end{aligned}
$$

3.2.1. Normalization and correlations. Following from equation (3.10), the normalization is

$$
N_{2}=4-2 \mu_{11}-2 \mu_{22}+\operatorname{det}[\boldsymbol{\mu}] .
$$

Defining the matrices $\widetilde{\mathbf{n}}=\left(2 \mathbf{I}-\boldsymbol{\mu}^{T}\right)^{-1}$ and $\mathbf{n}=\mathbf{I}-\widetilde{\mathbf{n}}$, where $\mathbf{I}$ is the $2 \times 2$ identity matrix, we can write the normalized two-mode Gaussian as

$$
\begin{aligned}
\widehat{\Lambda}_{2}(\mathbf{n})= & \operatorname{det}[\widetilde{\mathbf{n}}]: \exp \left[-\widehat{\boldsymbol{b}}^{\dagger}\left(2 \mathbf{I}-\widetilde{\mathbf{n}}^{-T}\right) \widehat{\boldsymbol{b}}\right]: \\
= & \operatorname{det}[\widetilde{\mathbf{n}}]|00\rangle\left\langle 00\left|+\left(n_{11} \widetilde{n}_{22}+n_{12} n_{21}\right)\right| 10\right\rangle\left\langle 10\left|+\left(n_{22} \widetilde{n}_{11}+n_{12} n_{21}\right)\right| 01\right\rangle\langle 01| \\
& +\operatorname{det}[\mathbf{n}]|11\rangle\left\langle 11\left|+n_{21}\right| 10\right\rangle\left\langle 01\left|+n_{12}\right| 01\right\rangle\langle 10| .
\end{aligned}
$$

If $\mathbf{n}$ is a Hermitian matrix, then the two-mode Gaussian corresponds to the density matrix of a mixture of states of different total number, with coherences $n_{12}=n_{21}^{*}$ between states of the same total number.

Normally ordered first-order correlations of the Gaussian operators correspond to elements of $\mathbf{n}$ :

$$
\left\langle\widehat{b}_{i}^{\dagger} \widehat{b}_{j}\right\rangle_{\widehat{\Lambda}} \equiv \operatorname{Tr}\left[\widehat{b}_{i}^{\dagger} \widehat{b}_{j} \widehat{\Lambda}_{2}\right]=n_{i j}
$$

and higher order correlations reduce to products of first-order averages, for example,

$$
\left\langle\widehat{b}_{1}^{\dagger} \widehat{b}_{1} \widehat{b}_{2}^{\dagger} \widehat{b}_{2}\right\rangle_{\widehat{\Lambda}}=n_{11} n_{22}-n_{12} n_{21} \text {. }
$$


This kind of factorisation of higher order correlations could be taken as the defining characteristic of a Gaussian state, and more generally, Gaussian operators, rather than the more formal operator definition given by equation (3.9). In other words, Gaussian operators have both a Gaussian form and generate Gaussian statistics.

We emphasize here that the correlations defined in equation (3.13) are only physical correlations when $\widehat{\Lambda}$ is a physical density matrix, since the Gaussian operators can also have unphysical correlations when $n_{i j}$ is not Hermitian.

Because the matrix $\mathbf{n}$ is Hermitian for the special case of a Gaussian that is a density operator, it can be diagonalized in these cases, corresponding to a change of single-particle basis. In this diagonalized basis, since the coherences are zero, the density operator is a mixture of number states, totally characterized by average occupation numbers. In other words, these Gaussian operators correspond to two-mode thermal states.

3.2.2. Completeness. We wish to show first that any number-conserving two-mode density matrix can be expanded in terms of Gaussian operators and second that this can be done with positive expansion coefficients. The first result follows if we can represent all the number-state projectors between states of the same total number using Gaussian operators. By inspection of equation (3.12) above, we find that

$$
\begin{aligned}
& |00\rangle\langle 00|=\widehat{\Lambda}_{2}(\mathbf{0}) \\
& |10\rangle\langle 10|=\widehat{\Lambda}_{2}\left(\left[\begin{array}{ll}
1 & 0 \\
0 & 0
\end{array}\right]\right) \\
& |01\rangle\langle 01|=\widehat{\Lambda}_{2}\left(\left[\begin{array}{ll}
0 & 0 \\
0 & 1
\end{array}\right]\right) \\
& |11\rangle\langle 11|=\widehat{\Lambda}_{2}(\mathbf{I}) \\
& n_{21}|10\rangle\langle 01|=\widehat{\Lambda}_{2}\left(\left[\begin{array}{cc}
n_{1} & 0 \\
n_{21} & n_{2}
\end{array}\right]\right)-\widehat{\Lambda}_{2}\left(\left[\begin{array}{cc}
n_{1} & 0 \\
0 & n_{2}
\end{array}\right]\right) \\
& n_{12}|01\rangle\langle 10|=\widehat{\Lambda}_{2}\left(\left[\begin{array}{cc}
n_{1} & n_{12} \\
0 & n_{2}
\end{array}\right]\right)-\widehat{\Lambda}_{2}\left(\left[\begin{array}{cc}
n_{1} & 0 \\
0 & n_{2}
\end{array}\right]\right) .
\end{aligned}
$$

Thus, the two-mode Gaussians form a complete operator basis for all number-conserving density matrices.

3.2.3. Positivity. Not only is the two-mode Gaussian a complete representation, but it is also a positive one: any two-mode number-conserving density operator can be written as a positive distribution over Gaussian operators. To see this, note that while the expression for the projection operators, equation (3.15), includes terms with negative coefficients, the projectors involved are the off-diagonal ones. Since density matrices are positive definite, off-diagonal projectors can only occur in combination with diagonal projectors. We take this into account in what follows.

Any two-mode density operator can be expanded into number-state projector operators as follows:

$$
\widehat{\rho}=\sum_{\vec{n}} \sum_{\vec{n}^{\prime}} \rho_{\vec{n}, \vec{n}^{\prime}}|\vec{n}\rangle\left\langle\vec{n}^{\prime}\right|
$$

where $\vec{n}$ and $\vec{n}^{\prime}$ are vectors of integer occupation numbers: $\vec{n}=\left(n_{1}, n_{2}\right), \vec{n}^{\prime}=\left(n_{1}^{\prime}, n_{2}^{\prime}\right)$. Here, $\rho_{\vec{n}, \vec{n}^{\prime}}=0$ if $\sum_{j} n_{j} \neq \sum_{j} n_{j}^{\prime}$, because of total-number conservation. Using the relations in 
equation (3.15), we can write the density operator as

$\widehat{\rho}=\sum_{\vec{n}} \frac{1}{2} \rho_{\vec{n}, \vec{n}}\left[\widehat{\Lambda}_{2}\left(\left[\begin{array}{cc}n_{1} & 2 \rho_{(01),(10)} \\ 0 & n_{2}\end{array}\right]\right)+\widehat{\Lambda}_{2}\left(\left[\begin{array}{cc}n_{1} & 0 \\ 2 \rho_{(10),(01)} & n_{2}\end{array}\right]\right)\right]$.

Since the diagonal coefficients $\rho_{\vec{n}, \vec{n}}$ are positive and sum to 1 , the Gaussian operators form the basis of a probabilistic representation of any two-mode density operator.

While any two-mode number-conserving density matrix can be expanded in the form in equation (3.17), there are often more direct representations. For example, as equation (3.20) shows, the entangled state $|\phi\rangle=\alpha|10\rangle+\beta|01\rangle$ can be represented by just one term, rather than the four terms that result from equation (3.17).

3.2.4. Correlation and entanglement. We first note that any uncorrelated product of numberstate mixtures can be represented exactly

$$
\begin{aligned}
\widehat{\rho}_{n_{1}} \otimes \widehat{\rho}_{n_{2}} & \equiv\left\{\tilde{n}_{1}|0\rangle\left\langle 0\left|+n_{1}\right| 1\right\rangle\langle 1|\right\} \otimes\left\{\tilde{n}_{2}|0\rangle\left\langle 0\left|+n_{2}\right| 1\right\rangle\langle 1|\right\} \\
& =\widehat{\Lambda}_{2}\left(\left[\begin{array}{cc}
n_{1} & 0 \\
0 & n_{2}
\end{array}\right]\right) .
\end{aligned}
$$

As well as these uncorrelated mixtures, the Gaussian basis can also be used to represent a mixture with correlations between the modes, this time as a sum (with positive weights) of two terms:

$$
A|00\rangle\langle 00|+B| 11\rangle\langle 11|=A \widehat{\Lambda}_{2}(\mathbf{0})+B \widehat{\Lambda}_{2}(\mathbf{I}) .
$$

Importantly, superpositions of number states, corresponding to entangled states, can also be represented, subject to total-number conservation. For example, the density matrix corresponding to the state $|\phi\rangle=\alpha|10\rangle+\beta|01\rangle$ is

$$
\begin{aligned}
|\phi\rangle\langle\phi| & =|\alpha|^{2}|10\rangle\left\langle 10\left|+\alpha \beta^{*}\right| 10\right\rangle\left\langle 01\left|+\alpha^{*} \beta\right| 01\right\rangle\left\langle\left. 10|+| \beta\right|^{2} \mid 01\right\rangle\langle 01| \\
& =\widehat{\Lambda}_{2}\left(\left[\begin{array}{cc}
|\alpha|^{2} & \alpha^{*} \beta \\
\alpha \beta^{*} & |\beta|^{2}
\end{array}\right]\right) .
\end{aligned}
$$

Thus, the number-conserving Gaussian operators even include entangled density matrices, making them a powerful tool for representing highly correlated states.

This property of being able to represent such Bell-like entangled states with the Hermitian subset of Gaussian operators is different to the case of Gaussian expansions for bosons. A typical example of this type of non-classical representation is the positive-P representation $[7,8]$, which must use a non-Hermitian basis to obtain a positive distribution that can represent all two-mode density matrices, such as those that violate a Bell inequality [22].

\subsection{Two-mode squeezed Gaussian operators}

Equation (3.9) does not represent the most general two-mode Gaussian form, as the quadratic form does not yet include terms such as $\widehat{b}_{1} \widehat{b}_{2}$. Incorporating such anomalous products, we can write the most general Gaussian operator in normalized form as

$$
\begin{aligned}
\widehat{\Lambda}_{2}^{(u)}\left(\boldsymbol{\mu}, \xi, \xi^{+}\right) & =: \exp \left[-\sum_{i, j=1}^{2} \mu_{i j} \widehat{b}_{i}^{\dagger} \widehat{b}_{j}-\xi^{+} \widehat{b}_{1} \widehat{b}_{2}-\xi \widehat{b}_{2}^{\dagger} \widehat{b}_{1}^{\dagger}\right]: \\
& =1-\sum_{i, j=1}^{2} \mu_{i j} \widehat{b}_{i}^{\dagger} \widehat{b}_{j}-\xi \widehat{b}_{2}^{\dagger} \widehat{b}_{1}^{\dagger}-\xi^{+} \widehat{b}_{1} \widehat{b}_{2}+\left(\operatorname{det}[\boldsymbol{\mu}]+\xi \xi^{+}\right) \widehat{b}_{1}^{\dagger} \widehat{b}_{2}^{\dagger} \widehat{b}_{2} \widehat{b}_{1},
\end{aligned}
$$


where $\xi$ and $\xi^{+}$are independent complex numbers. The two additional operator terms in the expansion, $\widehat{b}_{2}^{\dagger} \widehat{b}_{1}^{\dagger}$ and $\widehat{b}_{1} \widehat{b}_{2}$, are projectors between states of different total number:

$$
\widehat{b}_{1} \widehat{b}_{2}=-|00\rangle\left\langle 11\left|, \quad \widehat{b}_{2}^{\dagger} \widehat{b}_{1}^{\dagger}=-\right| 11\right\rangle\langle 00|,
$$

which are the kinds of coherences that appear in the density matrices of two-mode squeezed states.

3.3.1. Normalization and correlations. The normalization of the squeezed Gaussian is

$$
N_{2}=4-2 \mu_{11}-2 \mu_{22}+\operatorname{det}[\boldsymbol{\mu}]+\xi \xi^{+} \text {. }
$$

To incorporate this into a normalized Gaussian, we redefine $\mathbf{n}$ and $\widetilde{\mathbf{n}}$ such that

$$
\widetilde{\mathbf{n}}=\frac{N_{2}}{N_{2}-\xi \xi^{+}}\left(2 \mathbf{I}-\boldsymbol{\mu}^{T}\right)^{-1},
$$

and introduce rescaled squeezing parameters $m=-\xi / N_{2}, m^{+}=-\xi^{+} / N_{2}$. The normalized form is then

$\widehat{\Lambda}_{2}\left(\mathbf{n}, m, m^{+}\right)=\left(\operatorname{det}[\widetilde{\mathbf{n}}]+m m^{+}\right): \exp \left[\left(\widehat{\boldsymbol{b}}^{\dagger} \widehat{\boldsymbol{b}}^{T}\right)\left(\underline{\underline{I}}-\underline{\underline{\sigma}}^{-1} / 2\right)\left(\begin{array}{c}\widehat{\boldsymbol{b}} \\ \widehat{\boldsymbol{b}}^{\dagger T}\end{array}\right)\right]:$

where the $4 \times 4$ matrices $\underline{\underline{I}}$ and $\underline{\underline{\sigma}}$ are defined to be

$\underline{I}=\left[\begin{array}{cccc}-1 & 0 & 0 & 0 \\ 0 & -1 & 0 & 0 \\ 0 & 0 & 1 & 0 \\ 0 & 0 & 0 & 1\end{array}\right], \quad \underline{=}=\left[\begin{array}{cccc}-\tilde{n}_{11} & -\tilde{n}_{21} & 0 & m \\ -\tilde{n}_{12} & -\tilde{n}_{22} & -m & 0 \\ 0 & -m^{+} & \tilde{n}_{11} & \tilde{n}_{12} \\ m^{+} & 0 & \tilde{n}_{21} & \tilde{n}_{22}\end{array}\right]$.

In terms of number-state projectors, the normalized Gaussian is a generalisation of the number-conserving case, but with the additional non-number-conserving projectors:

$$
\begin{aligned}
\widehat{\Lambda}_{2}(\underline{\sigma})= & \left(\operatorname{det}[\widetilde{\mathbf{n}}]+m m^{+}\right)|00\rangle\left\langle 00\left|+\left(n_{11} \tilde{n}_{22}+n_{12} n_{21}-m m^{+}\right)\right| 10\right\rangle\langle 10| \\
& +\left(n_{22} \widetilde{n}_{11}+n_{12} n_{21}-m m^{+}\right)|01\rangle\left\langle 01\left|+\left(\operatorname{det}[\mathbf{n}]+m m^{+}\right)\right| 11\right\rangle\langle 11| \\
& +n_{21}|10\rangle\left\langle 01\left|+n_{12}\right| 01\right\rangle\langle 10|-m| 11\rangle\left\langle 00\left|-m^{+}\right| 00\right\rangle\langle 11| .
\end{aligned}
$$

In addition to the normal correlations of equation (3.13), the squeezed Gaussians can also have nonzero anomalous correlations, which are just equal to the new variables $m$ and $m^{+}$:

$$
\left\langle\widehat{b}_{1} \widehat{b}_{2}\right\rangle_{\widehat{\Lambda}}=m, \quad\left\langle\widehat{b}_{2}^{\dagger} \widehat{b}_{1}^{\dagger}\right\rangle_{\widehat{\Lambda}}=m^{+} .
$$

These correlations imply that for $\widehat{\Lambda}_{2}\left(\mathbf{n}, m, m^{+}\right)$to be a density matrix, $m$ and $m^{+}$must be complex conjugate. The second-order correlation generalizes to

$$
\left\langle\widehat{b}_{1}^{\dagger} \widehat{b}_{1} \widehat{b}_{2}^{\dagger} \widehat{b}_{2}\right\rangle_{\widehat{\Lambda}}=n_{11} n_{22}-n_{12} n_{21}+m m^{+},
$$

which again corresponds to the decorrelation that occurs in a state with Gaussian statistics.

3.3.2. Completeness. From equation (3.27), it follows that the squeezed Gaussians provide a complete two-mode fermionic basis, not only for the number-conserving subspace, but also for all states containing superpositions of states whose difference in total number is even. To see this, note that the projectors between the $|00\rangle$ and $|11\rangle$ number states can be written 
explicitly in terms of the Gaussian operators as

$$
\begin{aligned}
& m|11\rangle\langle 00|=\widehat{\Lambda}_{2}(\mathbf{n},-m, 0)-\widehat{\Lambda}_{2}(\mathbf{n}, 0,0), \\
& m^{+}|00\rangle\langle 11|=\widehat{\Lambda}_{2}\left(\mathbf{n}, 0,-m^{+}\right)-\widehat{\Lambda}_{2}(\mathbf{n}, 0,0),
\end{aligned}
$$

for any $\mathbf{n}$. These projectors, together with those of equation (3.15), span the complete Hilbert space of density matrices in question.

3.3.3. Positivity. Just as for the number-conserving case, we can write any physical density operator as a positive distribution over Gaussian operators:

$$
\begin{aligned}
\widehat{\rho}= & \sum_{\vec{n}} \sum_{\vec{n}^{\prime}} \rho_{\vec{n}, \vec{n}^{\prime}}|\vec{n}\rangle\left\langle\vec{n}^{\prime}\right| \\
= & \sum_{\vec{n}} \frac{1}{2} \rho_{\vec{n}, \vec{n}}\left[\widehat{\Lambda}_{2}\left(\left(\begin{array}{cc}
n_{1} & 2 \rho_{(01),(10)} \\
0 & n_{2}
\end{array}\right),-2 \rho_{(11,00)}, 0\right)\right. \\
& \left.+\widehat{\Lambda}_{2}\left(\left[\begin{array}{cc}
n_{1} & 0 \\
2 \rho_{(10),(01)} & n_{2}
\end{array}\right], 0,-2 \rho_{(00,11)}\right)\right] .
\end{aligned}
$$

Thus, the Gaussians form the basis of a probabilistic representation of any physical two-mode density operator. The ability to represent physical density matrices with the Gaussian basis, either as a single element or by a positive distribution over basis elements, is important for calculating dynamical simulations of quantum systems via probabilistic methods.

3.3.4. Entanglement. Again, these types of Gaussians can directly correspond to entangled density matrices, without having to consider expansions over several elements. But now superpositions of different total number can be represented. As an example of these additional kinds of physical states that the squeezed Gaussians can represent, consider the entangled non-number-conserving superposition state: $|\psi\rangle=[|00\rangle+|11\rangle] / \sqrt{2}$. This can be represented by a single Gaussian:

$$
\begin{aligned}
|\psi\rangle\langle\psi| & =\frac{1}{2}[|00\rangle\langle 00|+| 00\rangle\langle 11|+| 11\rangle\langle 00|+| 11\rangle\langle 11|] \\
& =\widehat{\Lambda}_{2}\left(\frac{1}{2} \mathbf{I},-\frac{1}{2},-\frac{1}{2}\right) .
\end{aligned}
$$

Hence, the more general type of Gaussian basis element considered here is considerably more powerful for representing entangled and correlated states than the number-conserving basis set.

\section{Multi-mode decomposition of Fermi systems}

So far we have considered only one- and two-mode systems, to illustrate the basic physical properties of Gaussian operators. As we saw, the Gaussian operators could easily be written in terms of number-state projectors. The power of the Gaussian operators as a basis in its own right becomes apparent for multi-mode systems, for which the number-state basis eventually becomes unusable due to exponential complexity: the Hilbert space dimension scales as $2^{M}$ for an $M$-mode Fermi system.

Before defining the general Gaussian basis for a system with many degrees of freedom, we define some mathematical notation and conventions that will be of subsequent use. Because we will always consider the general $M$-code case from here on, we will write $\widehat{\Lambda}$ without the subscript $M$, for notational simplicity. As before, we define $\widehat{b}$ as a column vector of the $M$ annihilation operators and $\widehat{\boldsymbol{b}}^{\dagger}$ as a row vector of the corresponding creation operators. We also 
introduce an extended column vector of all $2 M$ operators: $\underline{b}=\left(\widehat{\boldsymbol{b}}^{T}, \widehat{\boldsymbol{b}}^{\dagger}\right)^{T}$, with an adjoint row vector defined as $\underline{b}^{\dagger}=\left(\widehat{\boldsymbol{b}}^{\dagger}, \widehat{\boldsymbol{b}}^{T}\right)$. Writing out these in full, we get

$$
\underline{\underline{b}}=\left(\begin{array}{c}
\widehat{b}_{1} \\
\vdots \\
\widehat{b}_{M} \\
\widehat{b}_{1}^{\dagger} \\
\vdots \\
\widehat{b}_{M}^{\dagger}
\end{array}\right), \quad \underline{\hat{b}}^{\dagger}=\left(\widehat{b}_{1}^{\dagger}, \ldots, \widehat{b}_{M}^{\dagger}, \widehat{b}_{1}, \ldots, \widehat{b}_{M}\right) .
$$

Throughout the paper, we print length- $M$ vectors and $M \times M$ matrices in bold type, and index them where necessary with Latin indices: $j=1, \ldots, M$. Length- $2 M$ vectors we denote with an underline and $2 M \times 2 M$ matrices we denote with a double underline. These extended vectors and matrices are indexed where necessary with Greek indices: $\mu=1, \ldots, 2 M$. Note that an object such as $\underline{\widehat{b}} \underline{\underline{b}}^{\dagger}$ is a $2 M \times 2 M$ matrix:

$$
\underline{\underline{b}} \underline{\underline{b}}^{\dagger}=\left[\begin{array}{cc}
\widehat{\mathbf{b}}^{\dagger} & \widehat{\mathbf{b}}^{T} \\
\widehat{\mathbf{b}}^{\dagger T} \widehat{\mathbf{b}}^{\dagger} & \widehat{\mathbf{b}}^{\dagger T} \widehat{\mathbf{b}}^{T}
\end{array}\right],
$$

whereas $\underline{b}^{\dagger} \underline{\widehat{b}}=\sum_{k} \widehat{b}_{k}^{\dagger} \widehat{b}_{k}+\widehat{b}_{k} \widehat{b}_{k}^{\dagger}(=M)$ is a scalar. More general kinds of vectors are denoted with an arrow notation: $\vec{\mu}$.

For products of operators, we make use of normal and antinormal ordering concepts. Normal ordering, denoted by : ... : is defined as in the bosonic case, with all annihilation operators to the right of the creation operators, except that each pairwise reordering involved induces a sign change, e.g., $: \widehat{b}_{i} \widehat{b}_{j}^{\dagger}:=-\widehat{b}_{j}^{\dagger} \widehat{b}_{i}$. The sign changes are necessary so that the anticommuting natures of the Fermi operators can be accommodated without ambiguity. We define antinormal ordering similarly and denote it via curly braces: $\left\{\widehat{b}_{j}^{\dagger} \widehat{b}_{i}\right\}=-\widehat{b}_{i} \widehat{b}_{j}^{\dagger}$. More generally, we can define nested orderings, in which the outer ordering does not reorder the inner one. For example, $\left\{: \widehat{O} \widehat{b}_{j}^{\dagger}: \widehat{b}_{i}\right\}=-\widehat{b}_{i} \widehat{b}_{j}^{\dagger}: \widehat{O}:$, where $\widehat{O}$ is some operator.

The different orderings of pairs are, in block matrix form,

$$
\begin{gathered}
: \widehat{b}_{\mu} \widehat{b}_{\nu}^{\dagger}:=-: \widehat{b}_{\nu}^{\dagger} \widehat{b}_{\mu}:=\left[\begin{array}{cc}
-\widehat{b}_{j}^{\dagger} \widehat{b}_{i} & \widehat{b}_{i} \widehat{b}_{j} \\
\widehat{b}_{i}^{\dagger} \widehat{b}_{j}^{\dagger} & \widehat{b}_{i}^{\dagger} \widehat{b}_{j}
\end{array}\right], \\
\left\{\widehat{b}_{\mu} \widehat{b}_{\nu}^{\dagger}\right\}=-\left\{\widehat{b}_{\nu}^{\dagger} \widehat{b}_{\mu}\right\}=\left[\begin{array}{cc}
\widehat{b}_{i} \widehat{b}_{j}^{\dagger} & \widehat{b}_{i} \widehat{b}_{j} \\
\widehat{b}_{i}^{\dagger} \widehat{b}_{j}^{\dagger} & -\widehat{b}_{j} \widehat{b}_{i}^{\dagger}
\end{array}\right] .
\end{gathered}
$$

Note that this convention means that the relation between the two orderings is

$$
: \underline{b} \underline{b}^{\dagger}:=\underline{I}+\left\{\underline{\widehat{b} \widehat{b}^{\dagger}}\right\}
$$

where $\underline{I}$ is the constant matrix

$$
\underline{I} \equiv\left[\begin{array}{cc}
-\mathbf{I} & \mathbf{0} \\
\mathbf{0} & \mathbf{I}
\end{array}\right],
$$

in which $\mathbf{0}$ and $\mathbf{I}$ are the $M \times M$ zero and identity matrices, respectively.

When ordering products that contain a Gaussian operator $\widehat{\Lambda}$ (and later the density operator), we do not change the ordering of $\widehat{\Lambda}$ itself; the other operators are merely reordered around it. Thus, $\left\{: \widehat{\Lambda}_{i}^{\dagger}: \widehat{b}_{j}\right\}=-\widehat{b}_{j} \widehat{b}_{i}^{\dagger} \widehat{\Lambda}$. The different possible quadratic orderings 
containing a Gaussian operator can thus be written in matrix form as

$$
\begin{aligned}
& : \underline{\underline{b}} \hat{b}^{\dagger} \widehat{\Lambda}:=\left[\begin{array}{cc}
-\left(\widehat{\boldsymbol{b}}^{\dagger T} \widehat{\Lambda}^{\mathrm{b}} \widehat{\boldsymbol{b}}^{T}\right)^{T} & \widehat{\Lambda} \widehat{\boldsymbol{b}} \widehat{\boldsymbol{b}}^{T} \\
\widehat{\boldsymbol{b}}^{\dagger T} \widehat{\boldsymbol{b}}^{\dagger} \widehat{\Lambda} & \widehat{\boldsymbol{b}}^{\dagger T} \widehat{\Lambda} \widehat{\boldsymbol{b}}^{T}
\end{array}\right], \\
& \left\{\underline{\underline{b}} \underline{\underline{b}}^{\dagger} \widehat{\Lambda}\right\}=\left[\begin{array}{cc}
\widehat{\boldsymbol{b}} \widehat{\Lambda} \widehat{\boldsymbol{b}}^{\dagger} & \widehat{\boldsymbol{b}} \widehat{\boldsymbol{b}}^{T} \widehat{\Lambda} \\
\widehat{\Lambda} \widehat{\boldsymbol{b}}^{\dagger T} \widehat{\boldsymbol{b}}^{\dagger} & -\left(\widehat{\boldsymbol{b}} \widehat{\Lambda} \widehat{\boldsymbol{b}}^{\dagger}\right)^{T}
\end{array}\right], \\
& \left\{\underline{\underline{b}}: \underline{\underline{b}^{\dagger}} \widehat{\Lambda}:\right\}=\left[\begin{array}{cc}
\widehat{\boldsymbol{b}} \widehat{\boldsymbol{b}}^{\dagger} \widehat{\Lambda} & \widehat{\boldsymbol{b}} \hat{\Lambda} \widehat{\boldsymbol{b}}^{T} \\
-\left(\widehat{\boldsymbol{b}}^{\dagger} \widehat{\Lambda} \widehat{\boldsymbol{b}}^{\dagger}\right)^{T} & -\left(\widehat{\Lambda} \widehat{\boldsymbol{b}} \widehat{\boldsymbol{b}}^{\dagger}\right)^{T}
\end{array}\right], \\
& \left\{: \widehat{\Lambda} \underline{\underline{b}}: \underline{\underline{b}}^{\dagger}\right\}=\left[\begin{array}{cc}
\widehat{\Lambda} \widehat{\boldsymbol{b}} \widehat{\boldsymbol{b}}^{\dagger} & -\left(\widehat{\boldsymbol{b}} \widehat{\Lambda} \widehat{\boldsymbol{b}}^{T}\right)^{T} \\
\widehat{\boldsymbol{b}}^{\dagger T} \widehat{\Lambda} \widehat{\boldsymbol{b}}^{\dagger} & -\left(\widehat{\boldsymbol{b}} \widehat{\boldsymbol{b}}^{\dagger} \widehat{\Lambda}\right)^{T}
\end{array}\right] .
\end{aligned}
$$

\section{Normalized Gaussian operators}

\subsection{Definition}

We define a normalized Gaussian operator $\widehat{\Lambda}$ to be the most general Gaussian form of fermionic annihilation and creation operators, with zero displacement and unit trace. Using the extendedvector notation, we first write the normally ordered Gaussian in an unnormalized form as

$$
\widehat{\Lambda}^{(u)}\left(\boldsymbol{\mu}, \boldsymbol{\xi}, \boldsymbol{\xi}^{+}\right)=: \exp \left[\underline{\hat{b}^{\dagger}}\left(\underline{\underline{I}}-\underline{\underline{\sigma}}^{-1} / 2\right) \underline{\widehat{b}}\right]:,
$$

where we have introduced a new extended $2 M \times 2 M$ covariance matrix $\underline{\underline{\sigma}}$ defined in terms of $\mu, \xi, \xi^{+}$so that

$$
\underline{\underline{\sigma}}^{-1}=2 \underline{\underline{I}}+\left[\begin{array}{cc}
\mu & \xi \\
\xi^{+} & -\mu^{T}
\end{array}\right] .
$$

The introduction of the generalized covariance $\sigma$ allows the Gaussian operator to be written in a normalized form, using the results of appendix B, together with an explicit complex amplitude factor $\Omega$ :

$$
\widehat{\Lambda}(\vec{\lambda})=\Omega \operatorname{Pf}\left[\underline{\underline{\sigma_{A}}}\right]: \exp \left[\underline{\underline{b}}^{\dagger}\left(\underline{\underline{I}}-\underline{\underline{\sigma}}^{-1} / 2\right) \underline{\widehat{b}}\right]: .
$$

The normalization is one obvious difference with the conventional complex-number or bosonic Gaussian forms [9]. Chosen to ensure that $\operatorname{Tr} \widehat{\Lambda}=\Omega$, it contains the Pfaffian of an antisymmetric form $\sigma_{A}$ of the covariance. The choice of antisymmetrization is given in appendix $\mathrm{B}$; other choices will lead, in general, to additional sign factors. The square of the Pfaffian of an antisymmetric matrix is equal to its determinant and the determinant of

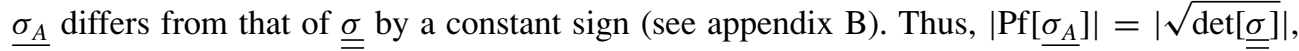
$\overline{\overline{\text { and }}}$ as we shall see, the relative phase between the two does not appear in later identities. The additional variable $\Omega$ plays the role of a weighting factor in the expansion that allows us to represent unnormalized density operators and to introduce stochastic gauges in the drift [23, 24].

We have chosen to impose on the covariance a type of generalized Hermitian antisymmetry, which can be written as $\underline{\underline{\sigma}}=-\underline{\underline{\sigma}}^{+}$, with the definition that

$$
\left[\begin{array}{ll}
\mathbf{a} & \mathbf{b} \\
\mathbf{c} & \mathbf{d}
\end{array}\right]^{+} \equiv\left[\begin{array}{ll}
\mathbf{d} & \mathbf{c} \\
\mathbf{b} & \mathbf{a}
\end{array}\right]^{T}
$$


It is this generalized antisymmetry that allows the covariance to be transformed into an explicitly antisymmetric matrix. The covariance can also be broken down into the physically significant $M \times M$ submatrices $\mathbf{n}, \mathbf{m}$ and $\mathbf{m}^{+}$:

$$
\underline{\underline{\sigma}}=\left[\begin{array}{cc}
-\widetilde{\mathbf{n}}^{T} & \mathbf{m} \\
\mathbf{m}^{+} & \widetilde{\mathbf{n}}
\end{array}\right]
$$

Here, $\mathbf{n}$ and $\widetilde{\mathbf{n}} \equiv 1-\mathbf{n}$ are complex matrices that correspond to the normal Green's functions for particles and holes, respectively, in many-body terminology, while $\mathbf{m}$ and $\mathbf{m}^{+}$are two independent antisymmetric complex matrices that correspond to anomalous Green's functions, as we will show in the next section. These quantities generalize the two-mode normal and anomalous correlations that were calculated previously to the $M$-mode case.

Thus, the total parametrization of a general Gaussian operator is

$$
\vec{\lambda}=\left(\Omega, \mathbf{n}, \mathbf{m}, \mathbf{m}^{+}\right)
$$

consisting of $1+p=1+M(2 M-1)$ parameters in all. However, for many systems, only a subset of all Gaussian operators is required for a complete representation of the density operator. One important subset is the set of generalized thermal states, for which $\mathbf{m}=\mathbf{m}^{+}=\mathbf{0}$. In this case, from appendix $\mathrm{B}$, and using the result that $2 \mathbf{I}-\boldsymbol{\mu}^{T}=\widetilde{\mathbf{n}}^{-1}$, we find the normalization factor to be $\operatorname{det}[\widetilde{\mathbf{n}}]$. The normalized thermal Gaussians therefore can be written as

$$
\widehat{\Lambda}(\vec{\lambda})=\Omega \operatorname{det}[\widetilde{\mathbf{n}}]: \exp \left[-\widehat{\mathbf{b}}^{\dagger}\left(2 \mathbf{I}-\widetilde{\mathbf{n}}^{-T}\right) \widehat{\mathbf{b}}\right]: .
$$

In order to use the Gaussian operator basis, we need to make use of a number of basic results. The proof of many of these can be established, as we show in appendix B, with Fermi coherent states and Grassmann algebra. The basics of these are given in appendix A. However, the final results do not contain any Grassmann variables.

\subsection{Trace properties}

Some basic traces are

$$
\operatorname{Tr}[\widehat{\Lambda}]=\Omega, \quad \operatorname{Tr}[\underline{b} \widehat{\Lambda}]=0, \quad \operatorname{Tr}\left[: \underline{\widehat{b}} \underline{\widehat{b}}^{\dagger} \widehat{\Lambda}:\right]=\Omega \underline{\underline{\sigma}} .
$$

The first of these is the normalization, proved as theorem 1 in appendix $B$. That the second is zero follows from the fact that the Gaussians are constructed from pairs of ladder operators and thus cannot correspond to a superposition of states whose total fermion numbers differ by one. The same result holds for the trace with any odd product. The third trace, proved as theorem 2 in appendix $\mathrm{B}$, allows us to calculate first-order correlations. In terms of the $M \times M$ submatrices, these become

$$
\operatorname{Tr}\left[\widehat{b}_{i}^{\dagger} \widehat{b}_{j} \widehat{\Lambda}\right]=\Omega n_{i j}, \quad \operatorname{Tr}\left[\widehat{b}_{i} \widehat{b}_{j} \widehat{\Lambda}\right]=\Omega m_{i j}, \quad \operatorname{Tr}\left[\widehat{b}_{i}^{\dagger} \widehat{b}_{j}^{\dagger} \widehat{\Lambda}\right]=\Omega m_{i j}^{+} .
$$

These results imply that for $\widehat{\Lambda}$ itself to correspond to a physical density matrix, $\mathbf{n}$ must be a Hermitian matrix, since $\left\langle\widehat{b}_{i}^{\dagger} \widehat{b}_{j}\right\rangle^{*}=\left\langle\widehat{b}_{j}^{\dagger} \widehat{b}_{i}\right\rangle$, and $\mathbf{m}^{+}$must be the Hermitian conjugate matrix of $\mathbf{m}$, since $\left\langle\widehat{b}_{i} \widehat{b}_{j}\right\rangle^{*}=\left\langle\widehat{b}_{j}^{\dagger} \widehat{b}_{i}^{\dagger}\right\rangle$. Physically, $\mathbf{n}$ gives the number, or normal correlations, and $\mathbf{m}$ and $\mathbf{m}^{+}$give the squeezing, or anomalous correlations.

Another restriction on $\widehat{\Lambda}$ being a physical density matrix that follows from equation (5.9) is that the eigenvalues of the matrix $\mathbf{n}$ lie in the interval $[0,1]$, because of the Pauli exclusion principle for fermions. Furthermore, the variance in the number correlations is

$$
\operatorname{var}\left\{\left\langle\widehat{b}_{i}^{\dagger} \widehat{b}_{j}\right\rangle\right\}=\left\langle\widehat{b}_{i}^{\dagger} \widehat{b}_{j}\right\rangle\left(1-\left\langle\widehat{b}_{i}^{\dagger} \widehat{b}_{j}\right\rangle\right),
$$

which implies that if all the eigenvalues of $\mathbf{n}$ are 0 or 1 , then $\widehat{\Lambda}$ is a number state, as the variance in number vanishes. If the eigenvalues are not limited to 0 or 1 , then $\widehat{\Lambda}$ corresponds 
to a mixture of number states in the eigenbasis and is thus a (possibly squeezed) thermal

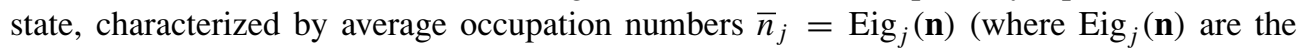
eigenvalues of $\mathbf{n}$ ) and a squeezing matrix $\mathbf{m}$.

A general Gaussian operator will not necessarily fulfil the Hermiticity condition and thus will not necessarily correspond to a physical state. However, the set of operators that do correspond to physical states is an important subclass, because a Gaussian operator expansion allows these states to be represented with a single element. The inclusion of nonphysical operators in the expansion, on the other hand, makes the Gaussian basis an (over)complete basis in which to expand a physical density operator of an arbitrary state. This is proved below for the general case. The overcompleteness of the Gaussian operators as a basis set has important implications for representing arbitrary states with a positive distribution function, a fact that we discuss in detail elsewhere [15].

\subsection{Completeness and positivity}

We next wish to show that the previous results on completeness and positivity obtained for one- and two-mode density matrix representations can be generalized to the multi-mode case. That is, we will prove that

- For any physical density matrix $\hat{\rho}$, a positive set of coefficients $P_{j}$ exists such that

$$
\hat{\rho}=\sum_{j} P_{j} \widehat{\Lambda}\left(\underline{\underline{\sigma}}^{(j)}\right) .
$$

This central result does not rely on utilizing the complex amplitudes $\Omega$, which are part of the most general Gaussian operator. If these were used, then positivity of the coefficients would be trivial, since these additional amplitudes could be used to absorb any phase or sign factors arising in the expansion. Instead, we wish to prove a much stronger result, that a positive expansion exists without any additional amplitude factors. This result is analogous to a similar result known for the positive-P bosonic representation [7, 8].

From the number-state basis of equation (2.2), the full set of possible fermionic manybody number states is the set $\{|\vec{n}\rangle\}$ where $\vec{n}$ is varied over all $2^{M}$ possible permutations. This defines a complete basis of $2^{2 M}$ number-state projectors for the set of all fermionic density operators. Even though not all the number-state projectors are Hermitian, we may nevertheless use this larger set of operators as a basis for the density matrices $\hat{\rho}$.

Next, we expand

$$
\begin{aligned}
\hat{\rho} & =\sum_{\vec{n}} \sum_{\vec{m}}|\vec{n}\rangle\langle\vec{n}|\hat{\rho}| \vec{m}\rangle\langle\vec{m}| \\
& =\sum_{\vec{n}} \sum_{\vec{m}} \rho_{\vec{n} \vec{m}}|\vec{n}\rangle\langle\vec{m}| \\
& =\sum_{\vec{n}} \sum_{\vec{m}} \hat{\rho}_{\vec{n} \vec{m}} .
\end{aligned}
$$

The positive definiteness of the density operator means in particular that diagonal density matrix elements are real and positive: $\rho_{\vec{n} \vec{n}}>0$. It is sufficient for completeness to prove that any elementary fermionic operator of form $\hat{\rho}_{\vec{n} \vec{m}}=\rho_{\vec{n} \vec{m}}|\vec{n}\rangle\langle\vec{m}|$ corresponds to a normalized Gaussian $\widehat{\Lambda}(\vec{\lambda})$, apart from a positive scaling factor. The demonstration proceeds by constructing limiting cases of Gaussians that correspond to each of the elementary components of the density matrix. As demonstrated in the one- and two-mode cases, such expansions are not unique, and generally one can obtain other more compact representations by combining diagonal and off-diagonal elements. 
To prove the elementary result, we proceed in three steps:

(1) Diagonal operators

First, the generic diagonal operator in the number basis is

$$
\begin{aligned}
\hat{\rho}_{\vec{n} \vec{n}} & =\rho_{\vec{n} \vec{n}}|\vec{n}\rangle\langle\vec{n}| \\
& =\rho_{\vec{n} \vec{n}} \prod_{i}\left|n_{i}\right\rangle_{i}\left\langle\left. n_{i}\right|_{i},\right.
\end{aligned}
$$

with a total occupation number

$$
N=\sum_{i} n_{i}
$$

Each diagonal multi-mode projector $|\vec{n}\rangle\langle\vec{n}|$ is simply an outer product of single-mode density matrices. From the single-mode example in section 3.1, each single-mode density matrix corresponds to a Gaussian as in equation (3.6). Thus, we see that a diagonal projector is exactly equal to a normalized Gaussian (we suppress the trivial arguments for simplicity):

$$
\begin{aligned}
|\vec{n}\rangle\langle\vec{n}| & =\prod_{i} \widehat{\Lambda}_{1}\left[n_{i}\right] \\
& =\widehat{\Lambda}_{M}[\mathbf{n}],
\end{aligned}
$$

where the matrix $\mathbf{n}$ is defined as $n_{i j}=n_{i} \delta_{i j}$.

Because $\rho_{\vec{n} \vec{n}}$ are real and positive, equation (5.13) shows that a positive Gaussian expansion exists for all diagonal density matrices:

$$
\widehat{\rho}_{\vec{n} \vec{n}}=\rho_{\vec{n} \vec{n}} \widehat{\Lambda}_{M}[\mathbf{n}] .
$$

In summary, a diagonal multi-mode projector $|\vec{n}\rangle\langle\vec{n}|$ is simply an outer product of single-mode density matrices, and hence corresponds exactly to a multi-mode normalized Gaussian.

\section{(2) Generalized thermal operators}

Next, consider off-diagonal projectors in the number-state expansion that conserve total number, i.e.,

$$
\widehat{\rho}_{\vec{n} \vec{m}}=\rho_{\vec{n} \vec{m}}|\vec{n}\rangle\langle\vec{m}|,
$$

for which

$$
\sum_{i} n_{i}=N=\sum_{i} m_{i}
$$

We show that any such component can be written as the limiting form of a number-conserving Gaussian, up to a positive scaling factor.

Let

$$
n_{i j}^{\prime}=\delta_{i j} \min \left\{n_{i}, m_{i}\right\},
$$

and define an off-diagonal Gaussian in terms of the diagonal normalized Gaussian:

$$
\widehat{\Lambda}^{(o)}\left(\boldsymbol{\mu}, \mathbf{n}^{\prime}\right)=: \prod_{i \neq j}\left[1-\mu_{i j} \hat{b}_{i}^{\dagger} \hat{b}_{j}\right] \widehat{\Lambda}\left[\mathbf{n}^{\prime}\right]:
$$

Now for every distinct mode $i$ with $\delta n_{i}=n_{i}-n_{i}^{\prime}=1$, one can define a corresponding distinct index $j(i)$ with $\delta m_{j}=m_{j}-m_{j}^{\prime}=1$. It follows from the minimum condition, equation (5.19), that $j(i) \neq i$, and from the number-conservation equation, equation (5.18), we have

$$
\sum_{i} \delta n_{i}=\sum_{i} \delta m_{i}
$$


Similarly, for every distinct pairs of indices $i, i^{\prime}$ with $\delta n_{i}=\delta n_{i^{\prime}}=1$, it follows that $j\left(i^{\prime}\right) \neq i$, since otherwise $\delta n_{i}=0$. The mapping therefore generates distinct pairs so that $j(i) \neq j\left(i^{\prime}\right) \Longleftrightarrow i \neq i^{\prime}$. Next, we note that this mapping is not a permutation of the set of modes $i$ with $\delta n_{i}=0$, since if it were the condition that $j\left(i^{\prime}\right) \neq i$ would be violated for some $i^{\prime}$. Similarly, the mapping is not a permutation of any subset of these modes. This means that the only non-vanishing terms in the normalization factor $\operatorname{det}[2 \mathbf{I}-\boldsymbol{\mu}]$ are the diagonal terms, which are already normalized.

Proceed by defining the resulting set of $\delta M \leqslant M$ pairs as $\Sigma=\{i, j\}$, and let

$$
\mu_{i j}(\varepsilon, \vec{n})=\sum_{\left\{i^{\prime}, j^{\prime}\right\} \in \Sigma} \frac{\mu \delta_{i i^{\prime}} \delta_{j j^{\prime}}}{-\varepsilon}
$$

where $\mu^{\delta M}=\rho_{\vec{n} \vec{m}}$, so that

$$
\begin{aligned}
\widehat{\Lambda}\left[\boldsymbol{\mu}(\varepsilon), \mathbf{n}^{\prime}\right] & =: \prod_{i \neq j}\left[1-\mu_{i j} \hat{b}_{i}^{\dagger} \hat{b}_{j}\right] \widehat{\Lambda}\left[\mathbf{n}^{\prime}\right]: \\
& =: \prod_{\{i, j\} \in \Sigma}\left[1+\frac{\mu \hat{b}_{i}^{\dagger} \hat{b}_{j}}{\varepsilon}\right]\left|\vec{n}^{\prime}\right\rangle\left\langle\vec{n}^{\prime}\right|: .
\end{aligned}
$$

Then consider the limit of $\varepsilon \rightarrow 0$, so that to leading order,

$$
\begin{aligned}
\widehat{\rho}_{\vec{n} \vec{m}} & =\mu^{\delta M}: \prod_{\{i, j\} \in \Sigma}\left[\hat{b}_{i}^{\dagger} \hat{b}_{j}\right]\left|\vec{n}^{\prime}\right\rangle\left\langle\vec{n}^{\prime}\right|: \\
& =\lim _{\varepsilon \rightarrow 0} \varepsilon^{\delta M} \widehat{\Lambda}\left[\boldsymbol{\mu}(\varepsilon), \mathbf{n}^{\prime}\right] .
\end{aligned}
$$

Again, we see that a positive expansion parameter is obtained.

(3) Squeezed operators

Finally, we consider the remaining elements of the density operator expansion, i.e., those squeezed projectors $\widehat{\rho}_{\vec{n}} \vec{m}$ for which

$$
N=\sum_{i} n_{i}=\sum_{i} m_{i}+2 S,
$$

where $S$ is an integer denoting the change in the number of fermion pairs between $\vec{n}$ and $\vec{m}$. We suppose that $S>0$, since the case of $S<0$ is obtained trivially by Hermitian conjugation. Let $n_{i}^{*}$ be obtained from $n_{i}$ by removing $2 S$ occupied sites, labelled as successive pairs $i, j$ belonging to a set $\Sigma^{*}$, so that $N^{*}=\sum_{i} n_{i}^{*}=\sum_{i} m_{i}$. The occupation numbers $n_{i}^{*}, m_{i}$ now define a generalized thermal density matrix component as previously, and hence equate to a limiting case of a Gaussian operator from equation (5.23).

Now define a squeezed off-diagonal Gaussian in terms of the thermal case, which we have already proved has a positive representation:

$$
\widehat{\Lambda}^{(s)}\left(\boldsymbol{\mu}(\varepsilon), \boldsymbol{\xi}(\varepsilon), \mathbf{n}^{\prime}\right)=\prod_{i>j}\left[1-\xi_{i j} \hat{b}_{i}^{\dagger} \hat{b}_{j}^{\dagger}\right] \widehat{\Lambda}\left[\boldsymbol{\mu}(\varepsilon), \mathbf{n}^{\prime}\right]
$$

where

$$
\xi_{i j}(\varepsilon)=\sum_{\left\{i^{\prime}, j^{\prime}\right\} \in \Sigma^{*}} \frac{\delta_{i i^{\prime}} \delta_{j j^{\prime}}}{-\varepsilon} .
$$

Then consider the limit of $\varepsilon \rightarrow 0$ as before, so that to leading order,

$$
\begin{aligned}
\widehat{\rho}_{\vec{n} \vec{m}} & =: \prod_{\{i, j\} \in \Sigma^{*}}\left[\hat{a}_{i}^{\dagger} \hat{a}_{j}^{\dagger}\right]\left|\vec{n}^{\prime}\right\rangle\langle\vec{m}|: \\
& =\lim _{\varepsilon \rightarrow 0} \varepsilon^{S+\delta M} \widehat{\Lambda}^{(s)}\left[\boldsymbol{\mu}(\varepsilon), \boldsymbol{\xi}(\varepsilon), \mathbf{n}^{\prime}\right] .
\end{aligned}
$$

A positive expansion parameter is obtained here as well, thus completing the proof. 


\subsection{Differential properties}

In order to use the Gaussian basis in a time-evolution problem, we need to be able to map the evolution of the density operator onto an evolution of the expansion coefficients $P_{j}$. To achieve this, one must be able to write the action of ladder operators on a Gaussian basis element in differential form.

We can differentiate the Gaussian operators with respect to their parameters to get

$$
\begin{aligned}
& \frac{\mathrm{d}}{\mathrm{d} \Omega} \widehat{\Lambda}=\frac{1}{\Omega} \widehat{\Lambda}, \\
& \frac{\mathrm{d}}{\mathrm{d} \underline{\underline{\sigma}}} \widehat{\Lambda}=\underline{\underline{\sigma}}^{-1} \widehat{\Lambda}-\underline{\underline{\sigma}}^{-1}: \underline{\underline{b}} \underline{\underline{b}}^{\dagger} \widehat{\Lambda}: \underline{\underline{\sigma}}^{-1},
\end{aligned}
$$

where the matrix derivative is defined as

$$
\left(\frac{\partial}{\partial \underline{\underline{\sigma}}}\right)_{\mu, v}=\frac{\partial}{\partial \sigma_{v \mu}}
$$

i.e., involving a transpose. When taking these derivatives, the antisymmetry of the covariance must be taken into account, as discussed in appendix B.

The expressions for the derivative can be inverted to obtain the important identities:

$$
\widehat{\Lambda}=\Omega \frac{\partial}{\partial \Omega} \widehat{\Lambda}, \quad: \underline{\underline{b}} \underline{\underline{b}} \hat{\Lambda}:=\underline{\underline{\sigma}} \widehat{\underline{\Lambda}}-\underline{\underline{\sigma}} \frac{\partial \widehat{\Lambda}}{\partial \underline{\underline{\sigma}}} \underline{\underline{\sigma}},
$$

and thus we can write the normally ordered action of any pair of operators on a Gaussian as a first-order derivative. As theorems 5 and 6 in appendix B show, there are analogous identities for antinormally ordered and mixed pairs:

$$
\left\{\underline{\underline{b}}: \underline{\underline{b}}^{\dagger} \widehat{\Lambda}:\right\}=-\underline{\underline{\sigma}} \widehat{\Lambda}-\underline{\underline{\sigma}} \frac{\partial \widehat{\Lambda}}{\partial \underline{\underline{\sigma}}} \underline{\underline{\sigma}}, \quad\{\underline{\underline{b}} \underline{\underline{b}} \dagger \hat{\Lambda}\}=-\underline{\underline{\sigma}} \widehat{\underline{\Lambda}}-\underline{\underline{\sigma}} \frac{\partial \widehat{\Lambda}}{\partial \underline{\underline{\sigma}}} \underline{\underline{\sigma}},
$$

where $\underline{\underline{\sigma}} \equiv \underline{I}-\underline{\underline{\sigma}}$.

For the subset of Gaussian operators that correspond to (generalized) thermal states, i.e., $\mathbf{m}^{+}=\mathbf{m}=\mathbf{0}$, we obtain a simpler set of differential identities:

$$
\begin{array}{rlrl}
\widehat{\boldsymbol{b}}^{\dagger T} \widehat{\boldsymbol{b}}^{T} \widehat{\Lambda} & =\mathbf{n} \widehat{\mathbf{\Lambda}}+\widetilde{\mathbf{n}} \frac{\partial \widehat{\Lambda}}{\partial \mathbf{n}} \mathbf{n}, & \widehat{\Lambda} \widehat{\boldsymbol{b}}^{\dagger T} \widehat{\boldsymbol{b}}^{T}=\mathbf{n} \widehat{\mathbf{\Lambda}}+\mathbf{n} \frac{\partial \widehat{\Lambda}}{\partial \mathbf{n}} \widetilde{\mathbf{n}}, \\
\widehat{\boldsymbol{b}}^{\dagger T} \widehat{\Lambda} \widehat{\boldsymbol{b}}^{T}=\widetilde{\mathbf{n}} \widehat{\Lambda}+\widetilde{\mathbf{n}} \frac{\partial \widehat{\Lambda}_{\widetilde{n}}}{\partial \mathbf{n}}, & \left(\widehat{\boldsymbol{b}} \widehat{\Lambda} \widehat{\boldsymbol{b}}^{\dagger}\right)^{T}=\mathbf{n} \widehat{\Lambda}-\mathbf{n} \frac{\partial \widehat{\Lambda}}{\partial \mathbf{n}} \mathbf{n} .
\end{array}
$$

The action of four ladder operators on a Gaussian operator can be obtained by applying the previous identities twice. Thus, in a Gaussian expansion, any two-body interaction term can be written as a second-order differential operator. As we show in detail elsewhere [15], this central result allows the evolving density operator to be mapped to a Fokker-Planck equation for the expansion coefficients, thus enabling a Monte Carlo sampling of the many-body quantum state.

\section{Conclusion}

In summary, we have introduced a generalized Gaussian operator basis, as a means of defining a non-orthogonal representation for correlated fermionic states. As special cases, the set of Gaussian operators include the density operators for thermal states and squeezed states. Thus the physics of the noninteracting Fermi gas and the BCS state is incorporated into 
the basis itself. Furthermore, the basis also includes more general operators, which ensure an overcompleteness that makes it possible to express any physical density operator as a probabilistic distribution over the Gaussian operators, without the need to use Grassmann algebra.

We have calculated the normalization and correlations, and proved completeness and positivity for the most general basis, and also for specific subsets, such as the numberconserving thermal basis. These results mean that the phase-space representation defined by the Gaussian operators can be used for first-principles simulations of many-body fermionic systems. The mapping from the quantum operator to the probabilistic $c$-number description is enabled by the Gaussian differential identities that have been derived here. The application of these identities will be dealt with elsewhere [15], when we consider the phase-space representation in more detail.

\section{Acknowledgment}

Funding for this research was provided by an Australian Research Council Centre of Excellence grant.

\section{Appendix A. Grassmann algebra}

This appendix introduces the basic concepts of noncommuting algebra and lists some results pertaining to Grassmann calculus and Fermi coherent states. These results are used in appendix B to establish important properties of the Gaussian operators. Proofs and further discussion of these results can be found in the literature [17-20].

Let $\boldsymbol{\alpha}$ be a vector of $M$ anticommuting (Grassmann) numbers, i.e.,

$$
\left[\alpha_{i}, \alpha_{j}\right]_{+}=0 .
$$

Since $\alpha_{j}^{2}=0$, any function of Grassmann numbers can be at most linear in any one of its arguments. Thus, for example, the single-mode exponential is

$$
\exp \left(\alpha_{j}\right)=1+\alpha_{j}
$$

and a multi-mode exponential, e.g., $\exp \left(\sum \alpha_{j}\right)$ will be an ordered product of such single-mode exponentials.

The Grassmann numbers anticommute with all Fermi annihilation and creation operators, but commute with $c$-numbers and bosonic operators.

\section{A.1. Grassmann calculus}

Differentiation of a single Grassmann variable is defined as

$$
\frac{\partial}{\partial \alpha_{i}} \alpha_{j}=\delta_{i j}
$$

with the derivative of products obtained by the Grassmann chain rule:

$$
\frac{\partial}{\partial \alpha_{j}}[f(\boldsymbol{\alpha}) g(\boldsymbol{\alpha})]= \begin{cases}\frac{\partial f}{\partial \alpha_{j}} g+f \frac{\partial g}{\partial \alpha_{j}}, & f \text { even } \\ \frac{\partial f}{\partial \alpha_{j}} g-f \frac{\partial g}{\partial \alpha_{j}}, & f \text { odd },\end{cases}
$$

i.e., the derivative operator also anticommutes. 
Grassmann integration is defined to be the same as differentiation, but written in a different way:

$$
\int \mathrm{d} \alpha_{j}=0, \quad \int \alpha_{j} \mathrm{~d} \alpha_{j}=1
$$

Multivariate integrals are ordered sequences of single-variable integrations, which can be written without ambiguity if the integration measure (as for a derivative) is also taken to be an anticommuting number. For an integral over all variables in a vector, we define the integration measure to be ordered in increasing numerical order: $\mathrm{d} \boldsymbol{\alpha} \equiv \mathrm{d} \alpha_{1} \cdots \mathrm{d} \alpha_{N}$.

Note that integrating a total derivative gives zero:

$$
\int \mathrm{d} \alpha_{j} \frac{\partial}{\partial \alpha_{j}} f=0
$$

This fact, coupled with the product rule, gives a result for partial integration:

$$
\int \mathrm{d} \alpha_{j} \frac{\partial f}{\partial \alpha_{j}} g= \begin{cases}-\int \mathrm{d} \alpha_{j} f \frac{\partial g}{\partial \alpha_{j}}, & f \text { even } \\ \int \mathrm{d} \alpha_{j} f \frac{\partial g}{\partial \alpha_{j}}, & f \text { odd. }\end{cases}
$$

One very useful result is the multivariate Gaussian integral:

$$
\int \exp \left(-\boldsymbol{\alpha}^{T} \mathbf{A} \boldsymbol{\alpha} / 2\right) \mathrm{d} \boldsymbol{\alpha}=\operatorname{Pf}[\mathbf{A}]
$$

for an antisymmetric matrix A of complex numbers. The Pfaffian is related to the determinant $(\operatorname{Pf}[\mathbf{A}])^{2}=\operatorname{det}[\mathbf{A}]$, and thus, apart from a sign change, has many of the same properties. For example, $\operatorname{Pf}\left[\mathbf{A}^{T}\right]=(-1)^{N} \operatorname{Pf}[\mathbf{A}]$ and $\left|\operatorname{Pf}\left[\mathbf{A}^{-1}\right]\right|=|1 / \operatorname{Pf}[\mathbf{A}]|$. Another useful type of Gaussian integral is

$$
\int \exp \left(-\boldsymbol{\beta}^{T} \mathbf{B} \boldsymbol{\alpha}\right) \prod_{j=1}^{M}\left(\mathrm{~d} \beta_{j} \mathrm{~d} \alpha_{j}\right)=\operatorname{det}[\mathbf{B}],
$$

where $\mathbf{B}$ is a square matrix of complex numbers and $\boldsymbol{\beta}$ and $\boldsymbol{\alpha}$ are the two independent Grassmann vectors. This second integral is in fact a special case of the first, except with $2 M$ total Grassmann variables.

\section{A.2. Grassmann coherent states}

For each Grassmann number $\alpha_{j}$ we can associate another Grassmann number, denoted as $\bar{\alpha}_{j}$, to play the role of a complex conjugate. This is formally regarded as an independent variable for calculus purposes. The conjugates $\bar{\alpha}_{j}$ anticommute with all other Grassmann variables. By use of such a complex Grassmann algebra, we can define a fermionic coherent state, which is formally an eigenstate of the annihilation operator:

$$
\begin{aligned}
\left|\alpha_{j}\right\rangle & =\left(1+\bar{\alpha}_{j} \alpha_{j}\right)^{-\frac{1}{2}}\left(|0\rangle+|1\rangle \alpha_{j}\right) \\
& =\exp \left(\widehat{b}_{j}^{\dagger} \alpha_{j}-\frac{1}{2} \bar{\alpha}_{j} \alpha_{j}\right)|0\rangle, \\
\left\langle\alpha_{j}\right| & =\left(1+\bar{\alpha}_{j} \alpha_{j}\right)^{-\frac{1}{2}}\left(\langle 0|+\bar{\alpha}_{j}\langle 1|\right) \\
& =\exp \left(\bar{\alpha}_{j} \widehat{b}_{j}-\frac{1}{2} \bar{\alpha}_{j} \alpha_{j}\right)\langle 0| .
\end{aligned}
$$


With these definitions, one can see that, like the bosonic coherent state, $\widehat{b}_{j}\left|\alpha_{j}\right\rangle=\alpha_{j}\left|\alpha_{j}\right\rangle$ and $\left\langle\alpha_{j}\right| \widehat{b}_{j}^{\dagger}=\left\langle\alpha_{j}\right| \alpha_{j}^{*}$ the fermionic coherent state can also be written as a (Grassmann) displacement from the vacuum:

$$
\left|\alpha_{j}\right\rangle=\exp \left(\widehat{b}_{j}^{\dagger} \alpha_{j}-\bar{\alpha}_{j} \widehat{b}_{j}\right)|0\rangle
$$

Multi-mode coherent states are products of the single-mode states:

$$
\begin{aligned}
|\boldsymbol{\alpha}\rangle & =\prod_{j=1}^{M}\left|\alpha_{j}\right\rangle \\
& =\exp \left[\left(\sum_{j=1}^{M} \widehat{b}_{j}^{\dagger} \alpha_{j}-\bar{\alpha}_{j} \widehat{b}_{j}\right)\right]|0\rangle .
\end{aligned}
$$

The inner product of two states is

$$
\left\langle\alpha_{i} \mid \alpha_{j}\right\rangle=\exp \left(\bar{\alpha}_{i} \alpha_{j}-\bar{\alpha}_{i} \alpha_{i} / 2-\bar{\alpha}_{j} \alpha_{j} / 2\right)
$$

and thus has two special cases:

$$
\left\langle\alpha_{i} \mid \alpha_{i}\right\rangle=1, \quad\left\langle-\alpha_{i} \mid . \alpha_{i}\right\rangle=\exp \left(-2 \bar{\alpha}_{i} \alpha_{i}\right)
$$

The usefulness of the coherent states lies in the fact that they form a complete set:

$$
\int \mathrm{d} \bar{\alpha}_{j} \mathrm{~d} \alpha_{j}\left|\alpha_{j}\right\rangle\left\langle\alpha_{j}|=| 0\right\rangle\langle 0|+| 1\rangle_{j}\langle 1|=I_{1}
$$

or, for the multi-mode case,

$$
\int \mathrm{d} \underline{\alpha}|\boldsymbol{\alpha}\rangle\langle\boldsymbol{\alpha}|=I_{M}
$$

where we have defined a $2 M$-variate integration measure as

$$
\mathrm{d} \underline{\alpha} \equiv \prod_{j=1}^{M}\left(\mathrm{~d} \bar{\alpha}_{j} \mathrm{~d} \alpha_{j}\right) .
$$

For later convenience, we define the alternative integration measure

$$
\mathrm{d} \bar{\alpha} \equiv \prod_{j=1}^{M}\left(\mathrm{~d} \alpha_{j} \mathrm{~d} \bar{\alpha}_{j}\right)=(-1)^{(M-1) M / 2} \mathrm{~d} \underline{\alpha} .
$$

Finally, we can express the trace of an arbitrary operator $\widehat{O}$ as

$$
\operatorname{Tr}[\widehat{O}]=\int \mathrm{d} \alpha \underline{\alpha}\langle-\boldsymbol{\alpha}|\widehat{O}| \boldsymbol{\alpha}\rangle .
$$

\section{Appendix B. Gaussian fermion operators}

We prove some useful results concerning the most general multi-mode Gaussian operator constructed from fermionic ladder operators. The proofs make use of the properties of Grassmann coherent states and anticommuting algebra, which are summarized in appendix A. However, the final results do not contain any Grassmann variables. The results establish the trace and differential properties of the Gaussian operators that are discussed in section 5. 


\section{B.1. General Gaussian operator}

In this appendix, we use the vector and ordering notations introduced in section 4 . We find it convenient to use an unnormalized Gaussian form of Fermi operators:

$$
\begin{aligned}
\widehat{\Lambda}^{(u)}(\underline{\mu}) & =: \exp \left[-\underline{\widehat{b}}^{\dagger} \underline{\underline{\mu}} \underline{\widehat{b}} / 2\right]: \\
& \equiv \sum_{n=0}^{\infty} \frac{1}{2^{n} n !}:\left(-\underline{\widehat{b}}^{\dagger} \underline{\underline{\mu}} \underline{\underline{b}}\right)^{n}: \\
& =: \prod_{\mu, \nu=1}^{2 M}\left(1-\frac{1}{2} \widehat{b}_{\mu}^{\dagger} \mu_{\mu \nu} \widehat{b}_{\nu}\right):
\end{aligned}
$$

where $\mu$ is a $2 M \times 2 M$ matrix of parameters, given by

$$
\underline{\underline{\mu}}=\left[\begin{array}{cc}
\boldsymbol{\mu} & \boldsymbol{\xi} \\
\boldsymbol{\xi}^{+} & -\boldsymbol{\mu}^{T}
\end{array}\right] .
$$

In terms of the covariance matrix $\underline{\underline{\sigma}}$, the unnormalized Gaussian is

$$
\widehat{\Lambda}^{(u)}(\underline{\underline{\sigma}})=: \exp \left[\underline{\underline{b}}^{\dagger}\left(\underline{\underline{I}}-\underline{\underline{\sigma}}^{-1} / 2\right) \underline{\underline{b}}\right]:
$$

where the relation between the two parametrizations is $\underline{\underline{\sigma}}=[\underline{\underline{\mu}}+2 \underline{\underline{I}}]^{-1}$ and where the diagonal matrix $\underline{I}$ is as defined in equation (4.5).

Because of the anticommuting property of fermions, we can impose upon $\underline{\underline{\sigma}}$ and $\underline{\underline{\mu}}$ a generalized antisymmetry: $\underline{\underline{\sigma}}=-{\underline{\underline{\sigma^{+}}}}, \underline{\underline{\mu}}=-\underline{\underline{\mu}}^{+}$, or in block matrix form,

$$
\begin{aligned}
& {\left[\begin{array}{ll}
\mathbf{a} & \mathbf{b} \\
\mathbf{c} & \mathbf{d}
\end{array}\right] \equiv-\underline{\underline{X}}\left[\begin{array}{ll}
\mathbf{a} & \mathbf{b} \\
\mathbf{c} & \mathbf{d}
\end{array}\right]^{T} \underline{\underline{X}}} \\
& =-\left[\begin{array}{ll}
\mathbf{d}^{T} & \mathbf{b}^{T} \\
\mathbf{c}^{T} & \mathbf{a}^{T}
\end{array}\right],
\end{aligned}
$$

where the constant matrix $\underline{\underline{X}}$ is defined as

$$
\underline{\underline{X}} \equiv\left[\begin{array}{ll}
\mathbf{0} & \mathbf{I} \\
\mathbf{I} & \mathbf{0}
\end{array}\right]
$$

When applied to the left of a matrix, $\underline{X}$ swaps the upper and lower halves; when applied to the right, it swaps the left and right halves. This structure means that the matrices $\underline{\sigma}$ and $\mu$ can be transformed into explicitly antisymmetric forms by certain permutations of rows $\overline{\bar{a}}$ d columns. For example, it follows immediately from equation (B.3) that interchanging left and right halves, or upper and lower halves, will generate an antisymmetric matrix. Alternatively, inserting each row in the lower half after the corresponding row in the upper half, and inserting each column in the right half before the corresponding row in the left half, generates the antisymmetric form

$$
\left[\begin{array}{ll}
\mathbf{a} & \mathbf{b} \\
\mathbf{c} & \mathbf{d}
\end{array}\right]_{A} \equiv\left[\begin{array}{ccccc}
b_{11} & a_{11} & \cdots & b_{1 M} & a_{1 M} \\
d_{11} & c_{11} & \cdots & d_{1 M} & c_{1 M} \\
\vdots & \vdots & \ddots & \vdots & \vdots \\
b_{M 1} & a_{M 1} & \cdots & b_{M M} & a_{M M} \\
d_{M 1} & c_{M 1} & \cdots & d_{M M} & c_{M M}
\end{array}\right] .
$$

With the covariance matrix antisymmetrized in this way, the Gaussian operator becomes

$$
\widehat{\Lambda}^{(u)}\left(\underline{\sigma}_{A}\right)=: \exp \left[\underline{\hat{b}}_{A}^{T}\left(\underline{I}_{A}-\underline{\sigma}_{A}^{-1} / 2\right) \underline{\underline{b}}_{A}\right]:
$$


where the vector of operators is now

$$
\widehat{\underline{b}}_{A}=\left(\begin{array}{c}
\widehat{b}_{1} \\
\widehat{b}_{1}^{\dagger} \\
\vdots \\
\widehat{b}_{M} \\
\widehat{b}_{M}^{\dagger}
\end{array}\right) .
$$

The generalized antisymmetry of $\underline{\underline{\sigma}}$ and $\underline{\underline{\mu}}$ has implications for matrix derivatives. Because each element of the matrix appears twice, we have $\partial \sigma_{\mu \nu} / \partial \sigma_{\theta \phi}=\delta_{\mu \theta} \delta_{\nu \phi}-X_{\mu \phi} X_{\nu \theta}$, where $\delta_{\mu \nu}$ is the Kronecker delta function. The extra terms here appear in the derivative of an inverse:

$$
\frac{\partial \sigma_{\mu \nu}^{-1}}{\partial \sigma_{\theta \phi}}=-\sigma_{\mu \theta}^{-1} \sigma_{\phi \nu}^{-1}+\sigma_{\mu \nu}^{-1} X_{\gamma \phi} X_{\theta \epsilon} \sigma_{\epsilon \nu}^{-1} .
$$

They also give an additional factor of 2 in the derivative of a determinant, thus

$$
\frac{\mathrm{d}}{\mathrm{d} \underline{\underline{\sigma}}} \sqrt{\operatorname{det} \underline{\underline{\sigma}}}=\sqrt{\operatorname{det} \underline{\underline{\sigma}}} \underline{\underline{\sigma}}^{-1},
$$

where we define the matrix derivative as

$$
\left(\frac{\mathrm{d}}{\mathrm{d} \underline{\underline{\sigma}}}\right)_{\mu \nu}=\frac{\mathrm{d}}{\mathrm{d} \sigma_{\nu \mu}},
$$

i.e., involving a transpose. The result (B.8) allows us to relate the derivatives with respect to $\underline{\underline{\sigma}}$ and $\underline{\underline{\mu}}$ :

$$
\frac{\mathrm{d} f}{\underline{\underline{d} \mu}}=\frac{\mathrm{d} f}{\mathrm{~d}^{-1}}=-\underline{\underline{\sigma}} \frac{\mathrm{d} f}{\underline{\underline{d}}} \underline{\underline{\sigma}} .
$$

\section{B.2. Normalization}

Theorem 1. The trace of the unnormalized Gaussian operator is equal to the Pfaffian of the inverse of the antisymmetrized covariance, i.e.,

$$
N \equiv \operatorname{Tr}\left[\widehat{\Lambda}^{(u)}(\underline{\underline{\sigma}})\right]=\operatorname{Pf}\left[\underline{\underline{\sigma}}_{A}^{-1}\right],
$$

where Pf stands for Pfaffian.

Proof. Because the Gaussian is in normally ordered form, it is straightforward to evaluate the trace with multi-mode Grassmann coherent states $|\boldsymbol{\alpha}\rangle$, using the Grassmann trace result of equation (A.19):

$$
\begin{aligned}
\operatorname{Tr}\left[\widehat{\Lambda}^{(u)}\right] & =\int\left\langle-\boldsymbol{\alpha}\left|\widehat{\Lambda}^{(u)}\right| \boldsymbol{\alpha}\right\rangle \mathrm{d} \underline{\alpha} \\
& =\int \exp \left[-\underline{\alpha}^{\dagger} \underline{\sigma}^{-1} \underline{\alpha} / 2\right] \mathrm{d} \bar{\alpha},
\end{aligned}
$$

where we have made use of the fact that, from the Grassmann inner product result of equation (A.13), $\langle-\boldsymbol{\alpha} \mid \boldsymbol{\alpha}\rangle=\exp (-2 \overline{\boldsymbol{\alpha}} \boldsymbol{\alpha})$. We have also changed variables: $\bar{\alpha}_{j} \rightarrow-\bar{\alpha}_{j}$, swapped the order of pairs in the integration measure and introduced $2 M$ vectors of Grassmann variables $\underline{\alpha}=\left(\boldsymbol{\alpha},(\overline{\boldsymbol{\alpha}})^{T}\right)$ and $\underline{\alpha}^{\dagger}=\left(\overline{\boldsymbol{\alpha}}, \boldsymbol{\alpha}^{T}\right)$. Changing to the antisymmetric form of the covariance $\underline{\underline{\sigma}}_{A}$ we obtain

$$
\operatorname{Tr}\left[\widehat{\Lambda}^{(u)}\right]=\int \exp \left[-\underline{\alpha}_{A}^{T} \underline{\underline{\sigma}}_{A}^{-1} \underline{\alpha}_{A} / 2\right] \mathrm{d} \bar{\alpha},
$$


where the reordered Grassmann vector is $\underline{\alpha}_{A}=\left(\alpha_{1}, \bar{\alpha}_{1}, \ldots, \alpha_{M}, \bar{\alpha}_{M}\right)^{T}$. Noting that the arrangement of elements in $\underline{\alpha}_{A}$ matches the order of integration, we can apply the Gaussian integral result [equation A.8)]:

$$
\operatorname{Tr}\left[\widehat{\Lambda}^{(u)}\right]=\operatorname{Pf}\left[\underline{\underline{\sigma}}_{A}^{-1}\right]
$$

Corollaries. Now the square of the Pfaffian of a matrix is equal to its determinant. Thus, to within $a \pm$ sign, the normalization is determined by the determinant of the covariance:

$$
\left(\operatorname{Tr}\left[\widehat{\Lambda}^{(u)}\right]\right)^{2}=\frac{1}{\operatorname{det}\left[\underline{\underline{\sigma}}_{A}\right]}=\frac{(-1)^{M}}{\operatorname{det}[\underline{\underline{\sigma}}]}=\frac{1}{\operatorname{det}[\underline{\underline{i}}]},
$$

and thus we may write the Gaussian operator in normalized form as

$$
\widehat{\Lambda}= \pm \Omega \sqrt{\operatorname{det}[\underline{\underline{\mathrm{i}}}]}: \exp \left[\underline{\underline{b}}^{\dagger}\left(\underline{\underline{I}}-\underline{\underline{\sigma}}^{-1} / 2\right) \underline{\underline{b}}\right]:
$$

where the choice of \pm sign is determined by $\sqrt{\operatorname{det}\left[\underline{\sigma}_{A}\right]} \operatorname{Pf}\left[\underline{\sigma}_{A}^{-1}\right]$.

This extra sign, or phase, which does not appear in the normalizations of the familiar complex-number or bosonic Gaussians, fortunately does not appear in any of the identities needed to make use of the Gaussian operators as a basis for a phase-space representation.

A specific case where the determinant appears is for the generalized thermal Gaussian without squeezing parameters, so that $\mathbf{m}=\mathbf{m}^{+}=0$. In this case, the normalization follows directly from the second Grassmann Gaussian integral identity, equation (A.9). Following the same procedure as before, we find that

$$
\operatorname{Tr}\left[\widehat{\Lambda}^{(u)}\right]=\operatorname{det}[2 \mathbf{I}-\boldsymbol{\mu}] .
$$

\section{B.3. First-order correlations}

Theorem 2. The fermionic Gaussian operator is completely characterized by its first-order correlations. In particular, the covariance matrix corresponds to the first-order correlations in normally ordered trace form, i.e.,

$$
\operatorname{Tr}\left[: \underline{\underline{b}} \underline{\mathrm{b}}^{\dagger} \widehat{\Lambda}:\right]=\Omega \underline{\underline{\sigma}},
$$

where $\underline{\widehat{b}} \underline{b}^{\dagger}$ is a matrix multiplication of two vectors, resulting in the $2 M \times 2 M$ matrix of equation (4.2).

Proof. We proceed as in the proof of theorem 1, by taking the trace of the unnormalized form using Grassmann coherent states and then changing variables, $\bar{\alpha}_{j} \rightarrow-\bar{\alpha}_{j}$,

$$
\begin{aligned}
\operatorname{Tr}\left[: \underline{\widehat{b}} \underline{\hat{b}}^{\dagger} \widehat{\Lambda}^{(u)}:\right] & =\int\left\langle-\boldsymbol{\alpha}\left|: \underline{\widehat{b}} \underline{\hat{b}}^{\dagger} \widehat{\Lambda}^{(u)}:\right| \boldsymbol{\alpha}\right\rangle \mathrm{d} \underline{\alpha} \\
& =\int \underline{\alpha \alpha} \underline{\alpha}^{\dagger} \exp \left[-\underline{\alpha}^{\dagger} \underline{\sigma}^{-1} \underline{\alpha} / 2\right] \mathrm{d} \bar{\alpha} .
\end{aligned}
$$

Next, we put the integral into the form (A.8):

$$
\begin{aligned}
\operatorname{Tr}\left[: \underline{\widehat{b}} \underline{b}^{\dagger} \widehat{\Lambda}^{(u)}:\right] & =\int-\left(\underline{\alpha}^{\dagger T} \underline{\alpha}^{T}\right)^{T} \exp \left[-\underline{\alpha}^{\dagger} \underline{\sigma}^{-1} \underline{\alpha} / 2\right] \mathrm{d} \bar{\alpha} \\
& =\frac{\mathrm{d}}{\mathrm{d} \underline{\underline{\sigma}}^{-1}} \int \exp \left[-\underline{\alpha}^{\dagger} \underline{\underline{\sigma}}^{-1} \underline{\alpha} / 2\right] \mathrm{d} \bar{\alpha}
\end{aligned}
$$


where in taking the derivative with respect to $\underline{\sigma}^{-1}$ we have taken account of the fact that each element of it appears twice, owing to its generalized antisymmetry. Evaluating the Grassmann integral and employing the determinant result, we get

$$
\begin{aligned}
\operatorname{Tr}\left[: \underline{\underline{b}} \underline{\underline{b}}^{\dagger} \widehat{\Lambda}^{(u)}:\right] & =\frac{\mathrm{d}}{\mathrm{d} \underline{\sigma}^{-1}}( \pm) \sqrt{\operatorname{det}\left[\underline{\mathrm{i}}^{-1}\right]} \\
& = \pm \sqrt{\operatorname{det}\left[\underline{\mathrm{i}}^{-1}\right]} \underline{\underline{\sigma}} .
\end{aligned}
$$

Finally, dividing through by the normalization in equation (B.12) gives the normalized result.

Corollaries. We can put equation (B.19) into a more familiar form by using the cyclic property of trace:

$$
\begin{aligned}
\operatorname{Tr}\left[: \underline{\underline{b}} \underline{\underline{b}}^{\dagger} \widehat{\Lambda}:\right] & =\operatorname{Tr}\left(\left[\begin{array}{cc}
-\left(\widehat{\mathbf{b}}^{\dagger T} \widehat{\Lambda} \widehat{\mathbf{b}}^{T}\right)^{T} & \widehat{\Lambda} \widehat{\mathbf{b}}^{T} \\
\widehat{\mathbf{b}}^{\dagger T} \widehat{\mathbf{b}}^{\dagger} \widehat{\Lambda} & \widehat{\mathbf{b}}^{\dagger T} \widehat{\Lambda} \widehat{\mathbf{b}}^{T}
\end{array}\right]\right) \\
& =\operatorname{Tr}\left(\left[\begin{array}{cc}
-\widehat{\mathbf{b}}^{\dagger} & \widehat{\mathbf{b}}^{T} \\
\widehat{\mathbf{b}}^{\dagger T} \widehat{\mathbf{b}}^{\dagger} & \left(\widehat{\mathbf{b}}^{\dagger}\right)^{T}
\end{array}\right] \widehat{\Lambda}\right) \\
& =\Omega\left[\begin{array}{cc}
-\widetilde{\mathbf{n}}^{T} & \mathbf{m} \\
\mathbf{m}^{+} & \widetilde{\mathbf{n}}
\end{array}\right] .
\end{aligned}
$$

We have defined the matrices $\mathbf{n}$ and $\widetilde{\mathbf{n}} \equiv \mathbf{I}-\mathbf{n}$ for the normal correlations of particles and holes, respectively, and the matrices $\mathbf{m}$ and $\mathbf{m}^{+}$for the squeezing, or anomalous, correlations, as follows:

$$
\mathbf{n}=\left\langle\widehat{\mathbf{b}}^{\dagger T} \widehat{\mathbf{b}}^{T}\right\rangle_{\widehat{\Lambda}}, \quad \mathbf{m}=\left\langle\widehat{\mathbf{b}}^{T}\right\rangle_{\widehat{\Lambda}}, \quad \mathbf{m}^{+}=\left\langle\widehat{\mathbf{b}}^{\dagger T} \widehat{\mathbf{b}}^{\dagger}\right\rangle_{\widehat{\Lambda}},
$$

where $\langle\widehat{O}\rangle_{\widehat{\Lambda}} \equiv \operatorname{Tr}[\widehat{O} \widehat{\Lambda}] \Omega$. Thus, we can write the covariance matrix in terms of the correlations as

$$
\underline{\underline{\sigma}}=\left[\begin{array}{cc}
-\widetilde{\mathbf{n}}^{T} & \mathbf{m} \\
\mathbf{m}^{+} & \widetilde{\mathbf{n}}
\end{array}\right],
$$

or inverting the relationship

$$
\left\langle: \underline{\underline{b}} \underline{\underline{b}}^{\dagger}:\right\rangle_{\widehat{\Lambda}}=\underline{\underline{I}} \underline{\underline{\sigma}} \underline{\underline{I}},
$$

where $\underline{\underline{\sigma}}=\underline{\underline{I}}-\underline{\underline{\sigma}}$.

\section{B.4. Higher order correlations}

One can calculate higher order correlations along similar lines, i.e., by expanding the trace in fermionic coherent states and using standard results for Gaussian integrals. The result is a sum over permutations of products of first-order correlations, with appropriate signs for fermion antisymmetry.

Theorem 3. An rth-order correlation of a Gaussian operator, in normally ordered trace form, factorizes into sums of first-order correlations as follows:

$\operatorname{Tr}\left[: \widehat{\mathrm{b}}_{\mu_{1}} \cdots \widehat{\mathrm{b}}_{\mu_{2 \mathrm{r}}} \widehat{\Lambda}:\right]=\Omega^{1-r} \sum_{P}(-1)^{P} \operatorname{Tr}\left[: \widehat{b}_{\nu_{1}} \widehat{b}_{\nu_{2}} \widehat{\Lambda}:\right] \times \cdots \times \operatorname{Tr}\left[: \widehat{b}_{v_{2 r-1}} \widehat{b}_{v_{2 r}} \widehat{\Lambda}:\right]$

where $v_{j}=\mu_{P(j)}$. The sum is over all $2 r ! /\left(2^{r} r\right.$ !) distinct pair permutations $P(1), \ldots, P(2 r)$ and the sign $(-1)^{P}$ is the parity of the permutation. 
Proof. We start by expressing the trace in terms of fermionic coherent states:

$$
\begin{aligned}
\operatorname{Tr}\left[: \widehat{b}_{\mu_{1}} \cdots \widehat{b}_{\mu_{2 r}} \widehat{\Lambda}:\right] & =\int\left\langle-\boldsymbol{\alpha}\left|: \widehat{b}_{\mu_{1}} \cdots \widehat{b}_{\mu_{2 r}} \widehat{\Lambda}:\right| \boldsymbol{\alpha}\right\rangle \underline{\mathrm{d}} \underline{\alpha} \\
& =\frac{\Omega}{N} \int \alpha_{\mu_{1}} \cdots \alpha_{\mu_{2 r}} \exp \left(-\underline{\alpha}^{\dagger} \underline{\sigma}^{-1} \underline{\alpha} / 2\right) \mathrm{d} \bar{\alpha} \\
& =\frac{\Omega}{N} \int \alpha_{A\left(\mu_{1}\right)} \cdots \alpha_{A\left(\mu_{2 r}\right)} \exp \left(-\underline{\alpha}_{A}^{T} \underline{\sigma}_{A}^{-1} \underline{\alpha}_{A} / 2\right) \mathrm{d} \bar{\alpha}
\end{aligned}
$$

where $A(\mu)$ is the permutation required to convert $\underline{\alpha}$ to $\underline{\alpha}_{A}$. The integral is now in standard form and we can thus apply the appropriate result from [19] to give

$$
\begin{aligned}
\operatorname{Tr}\left[: \widehat{b}_{\mu_{1}} \cdots \widehat{b}_{\mu_{2 r}} \widehat{\Lambda}:\right] & \left.=\Omega^{1-r} \sum_{P}(-1)^{P} \underline{\underline{\sigma}}_{A}\right\}_{A\left(v_{1}\right), A\left(v_{2}\right)} \times \cdots \times\left\{\underline{\underline{\sigma}}_{A}\right\}_{A\left(v_{2 r-1}\right), A\left(v_{2 r}\right)} \\
& =\Omega^{1-r} \sum_{P}(-1)^{P} \sigma_{v_{1} v_{2}^{\prime}} \cdots \sigma_{v_{2 r-1}, v_{2 r}^{\prime}},
\end{aligned}
$$

where $v_{j}=\mu_{P(j)}$ and where $v_{j}^{\prime}=\left(v_{j}+M\right) \bmod 2 M$. Finally, substituting in the result for first-order correlations on the right-hand side gives equation (B.27).

Examples. A general second-order correlation is of the form (for $\Omega=1$ )

$$
\operatorname{Tr}\left[: \widehat{b}_{\mu_{1}} \widehat{b}_{\mu_{2}} \widehat{b}_{\mu_{3}} \widehat{b}_{\mu_{4}} \widehat{\Lambda}:\right]=\sigma_{\mu_{1} \mu_{2}^{\prime}} \sigma_{\mu_{3} \mu_{4}^{\prime}}+\sigma_{\mu_{1} \mu_{4}^{\prime}} \sigma_{\mu_{2} \mu_{3}^{\prime}}+\sigma_{\mu_{1} \mu_{3}^{\prime}} \sigma_{\mu_{4} \mu_{2}^{\prime}} .
$$

Thus, for example, the normally ordered number-number correlations are

$$
\operatorname{Tr}\left[: \widehat{n}_{i} \widehat{n}_{j}: \widehat{\Lambda}\right]=n_{i i} n_{j j}-n_{i j} n_{j i}-m_{i j} m_{i j}^{+},
$$

i.e., containing the three terms expected for a state with Gaussian correlations.

Similarly, a third-order correlation is of the form (for $\Omega=1$ )

$$
\operatorname{Tr}\left[: \widehat{b}_{\mu_{1}} \ldots \widehat{b}_{\mu_{6}} \widehat{\Lambda}:\right]=\sum_{P}(-1)^{P} \sigma_{v_{1} v_{2}^{\prime}} \sigma_{\nu_{3} v_{4}^{\prime}} \sigma_{v_{5} v_{6}^{\prime}}
$$

where $v_{j}=\mu_{P(j)}$. Thus, the normally ordered triple correlations are

$$
\begin{aligned}
\operatorname{Tr}\left[: \widehat{n}_{i} \widehat{n}_{j} \widehat{n}_{k}: \widehat{\Lambda}\right] & =n_{i i} n_{j j} n_{k k}-n_{i i}\left(n_{j k} n_{k j}+m_{j k} m_{j k}^{+}\right)+n_{i j} n_{j k} n_{k i}-n_{j j}\left(n_{i k} n_{k i}+m_{i k} m_{i k}^{+}\right) \\
+ & n_{j i} n_{k j} n_{i k}-n_{k k}\left(n_{i j} n_{j i}+m_{i j} m_{i j}^{+}\right)+n_{i j} m_{i k} m_{j k}^{+}+n_{j i} m_{j k} m_{i k}^{+} \\
& +n_{j k} m_{j i} m_{k i}^{+}+n_{k j} m_{k i} m_{j i}^{+}+n_{k i} m_{k j} m_{i j}^{+}+n_{i k} m_{k j} m_{i j}^{+}
\end{aligned}
$$

again as expected for a state with Gaussian correlations.

\section{B.5. Normally ordered products}

Theorem 4. A normally ordered product of a pair of ladder operators and a Gaussian is equivalent to a first-order differential operator on the Gaussian, as follows:

$$
: \underline{\underline{b}} \underline{b}^{\dagger} \widehat{\Lambda}:=\underline{\underline{\sigma}} \widehat{\Lambda}-\underline{\underline{\sigma}} \frac{\partial \widehat{\Lambda}}{\partial \underline{\underline{\sigma}}} \underline{\underline{\sigma}} .
$$

Proof. The proof can be established easily without Grassmann algebra. We first take the derivative of the unnormalized Gaussian operator:

$$
\frac{\partial}{\partial \mu_{\mu \nu}} \widehat{\Lambda}^{(u)}(\underline{\underline{\mu}})=-: \widehat{b}_{\mu}^{\dagger} \widehat{\Lambda}^{(u)}\left(\underline{\underline{\mu})} \widehat{b}_{\nu}:\right. \text {. }
$$


We write this as a derivative with respect to the covariance matrix, using equation (B.11), and swap the pair of operators, to give

$$
\underline{\underline{\sigma}} \frac{\mathrm{d} \widehat{\Lambda}^{(u)}(\underline{\underline{\sigma}})}{\mathrm{d} \underline{\underline{\sigma}}} \underline{\underline{\sigma}}=-: \underline{\hat{b}} \underline{\hat{b}}^{\dagger} \widehat{\Lambda}^{(u)}(\underline{\underline{\sigma}}):
$$

Next, we take the derivative of the normalization, using equation (B.9),

$$
\begin{aligned}
\frac{\mathrm{d}}{\mathrm{d} \underline{\underline{\sigma}}} N & =\frac{\mathrm{d}}{\mathrm{d} \underline{\underline{\sigma}}}( \pm)(\operatorname{det}[\underline{\underline{\sigma}}])^{-\frac{1}{2}} \\
& =-N \underline{\underline{\sigma}}^{-1} .
\end{aligned}
$$

Combining both of these results, we find that the derivative of the normalized Gaussian is

$$
\begin{aligned}
\frac{\mathrm{d}}{\mathrm{d} \underline{\underline{\sigma}}} \widehat{\Lambda} & =-\Omega N^{-2} \widehat{\Lambda}^{(u)} \frac{\mathrm{d} N}{\mathrm{~d} \sigma}+\Omega N^{-1} \frac{\mathrm{d}}{\mathrm{d} \sigma} \widehat{\Lambda}^{(u)} \\
& =\underline{\underline{\sigma}}^{-1} \widehat{\Lambda}-\underline{\underline{\sigma}}^{-1}: \underline{\underline{b}} \underline{\underline{b}} \hat{\Lambda}: \underline{\underline{\sigma}}^{-1},
\end{aligned}
$$

whose inverse is the required result.

This result can also be proved by use of Grassmann coherent-state expansions, in similar manner to the proofs below for the products of different ordering.

\section{B.6. Mixed products}

Theorem 5. A product of mixed order of a pair of ladder operators and a Gaussian is equivalent to a first-order differential operator on the Gaussian, as follows:

$$
\{\underline{\underline{b}}: \underline{\underline{b}} \hat{\Lambda}:\}=-\underline{\underline{\sigma}} \widehat{\underline{\Lambda}}-\underline{\underline{\sigma}} \frac{\partial \widehat{\Lambda}}{\partial \underline{\underline{\sigma}}} \underline{\underline{\sigma}} .
$$

Proof. We first make use of the Fermi coherent-state completeness identity to replace all ladder operators by Grassmann integrals over coherent projection operators:

$$
\begin{aligned}
\left\{\underline{\widehat{b}}: \underline{\underline{b}}^{\dagger} \widehat{\Lambda}:\right\}= & \int \mathrm{d} \underline{\gamma} \mathrm{d} \underline{\beta} \mathrm{d} \underline{\alpha} \mathrm{d} \underline{\epsilon}|\gamma\rangle\langle\gamma|\{\underline{\underline{b}}:|\boldsymbol{\beta}\rangle\langle\boldsymbol{\beta}|\underline{\hat{b}} \hat{\Lambda}| \boldsymbol{\alpha}\rangle\langle\boldsymbol{\alpha}|:\}| \epsilon\rangle\langle\epsilon| \\
= & \frac{\Omega}{N} \int \mathrm{d} \underline{\gamma} \mathrm{d} \underline{\beta} \mathrm{d} \underline{\alpha} \mathrm{d} \underline{\epsilon}|\gamma\rangle\langle\epsilon| \exp \left[-\frac{1}{2}(\overline{\boldsymbol{\beta}}, \boldsymbol{\alpha}) \underline{\underline{\mu}}=\left(\frac{\boldsymbol{\alpha}}{\boldsymbol{\beta}}\right)\right] \\
& \times\left(\begin{array}{c}
\boldsymbol{\beta} \\
\overline{\boldsymbol{\alpha}}
\end{array}\right)(\overline{\boldsymbol{\beta}}, \boldsymbol{\alpha}) \exp \left[\bar{\gamma} \boldsymbol{\beta}+\overline{\boldsymbol{\alpha}} \epsilon-\overline{\boldsymbol{\alpha}} \boldsymbol{\alpha}-\overline{\boldsymbol{\beta}} \boldsymbol{\beta}-\frac{1}{2} \bar{\gamma} \boldsymbol{\gamma}-\frac{1}{2} \bar{\epsilon} \epsilon\right],
\end{aligned}
$$

where $\underline{\underline{\mu}} \equiv \underline{\underline{\mu}}+\underline{\underline{I}}$. We have used the result that the inner product of two coherent states is, from equation (A.13),

$$
\langle\boldsymbol{\beta} \mid \boldsymbol{\alpha}\rangle=\exp [\overline{\boldsymbol{\beta}} \boldsymbol{\alpha}-\overline{\boldsymbol{\beta}} \boldsymbol{\beta} / 2-\overline{\boldsymbol{\alpha}} \boldsymbol{\alpha} / 2] .
$$

Next, we employ integration by parts (equation (A.7)) to replace $\left(\frac{\beta}{\alpha}\right)$ by variables that appear in the Gaussian form:

$$
\begin{aligned}
\exp [-(\overline{\boldsymbol{\beta}}, \boldsymbol{\alpha}) & \underline{\tilde{\mu}} \\
= & \left.\left(\begin{array}{c}
\boldsymbol{\alpha} \\
\overline{\boldsymbol{\beta}}
\end{array}\right) / 2\right]\left[\left(\begin{array}{c}
\boldsymbol{\beta} \\
\overline{\boldsymbol{\alpha}}
\end{array}\right) \exp (-\overline{\boldsymbol{\alpha}} \boldsymbol{\alpha}-\overline{\boldsymbol{\beta}} \boldsymbol{\beta})\right](\overline{\boldsymbol{\beta}}, \boldsymbol{\alpha}) \\
& =\exp \left[-(\overline{\boldsymbol{\beta}}, \boldsymbol{\alpha}) \underline{\underline{\underline{\mu}}}\left(\begin{array}{l}
\boldsymbol{\alpha} \\
\overline{\boldsymbol{\beta}}
\end{array}\right) / 2\right]\left[\left(\begin{array}{c}
-\partial_{\overline{\boldsymbol{\beta}}} \\
\partial_{\boldsymbol{\alpha}}
\end{array}\right) \exp (-\overline{\boldsymbol{\alpha}} \boldsymbol{\alpha}-\overline{\boldsymbol{\beta}} \boldsymbol{\beta})\right](\overline{\boldsymbol{\beta}}, \boldsymbol{\alpha})
\end{aligned}
$$




$$
\begin{aligned}
\rightarrow & \exp (-\overline{\boldsymbol{\alpha}} \boldsymbol{\alpha}-\overline{\boldsymbol{\beta}} \boldsymbol{\beta})\left(\begin{array}{c}
\partial_{\overline{\boldsymbol{\beta}}} \\
-\partial_{\alpha}
\end{array}\right) \exp \left[-\frac{1}{2}(\overline{\boldsymbol{\beta}}, \boldsymbol{\alpha}) \underline{\underline{\mu}}\left(\frac{\boldsymbol{\alpha}}{\overline{\boldsymbol{\beta}}}\right)\right](\overline{\boldsymbol{\beta}}, \boldsymbol{\alpha}) \\
= & \exp (-\overline{\boldsymbol{\alpha}} \boldsymbol{\alpha}-\overline{\boldsymbol{\beta}} \boldsymbol{\beta})\left[\underline{\underline{I}} \underline{\underline{\mu}}\left(\begin{array}{c}
\boldsymbol{\alpha} \\
\overline{\boldsymbol{\beta}}
\end{array}\right)(\overline{\boldsymbol{\beta}}, \boldsymbol{\alpha})-\underline{\underline{I}}\right] \\
& \times \exp \left[-(\overline{\boldsymbol{\beta}}, \boldsymbol{\alpha}) \underline{\underline{\mu}}\left(\begin{array}{c}
\boldsymbol{\alpha} \\
\overline{\boldsymbol{\beta}}
\end{array}\right) / 2\right] .
\end{aligned}
$$

We can now express the result as a derivative of the unnormalized Gaussian operator with respect to $\underline{\underline{\mu}}$ :

$$
\begin{aligned}
\left\{\underline{\widehat{b}}: \underline{\hat{b}^{\dagger}} \widehat{\Lambda}:\right\}= & \frac{\Omega}{N} \int \mathrm{d} \underline{\gamma} \mathrm{d} \underline{\beta} \mathrm{d} \underline{\alpha} \mathrm{d} \underline{\epsilon}|\gamma\rangle\langle\epsilon| \exp \left[-\overline{\boldsymbol{\alpha}} \boldsymbol{\alpha}-\overline{\boldsymbol{\beta}} \boldsymbol{\beta}-\frac{1}{2} \bar{\gamma} \gamma-\frac{1}{2} \bar{\epsilon} \epsilon+\bar{\gamma} \boldsymbol{\beta}+\overline{\boldsymbol{\alpha}} \epsilon\right] \\
& \times\left[\underline{\underline{I}} \underline{\underline{\mu}} \frac{\mathrm{d}}{\mathrm{d}}-\underline{\underline{\mu}}=\underline{\underline{I}}\right] \exp \left[-\frac{1}{2}(\overline{\boldsymbol{\beta}}, \boldsymbol{\alpha}) \underline{\underline{\mu}}\left(\frac{\boldsymbol{\alpha}}{\overline{\boldsymbol{\beta}}}\right)\right] \\
= & \frac{\Omega}{N}\left[\underline{\underline{I}}=\frac{\mathrm{\mu}}{=} \frac{\mathrm{d}}{\mathrm{d} \underline{\mu}}-\underline{\underline{I}}\right] \widehat{\Lambda}^{(u)} .
\end{aligned}
$$

Finally, we can change variables to $\underline{\underline{\sigma}}=[\underline{\underline{\mu}}+\underline{\underline{I}}]^{-1}$ and use the result for the derivative of the normalization:

$$
\begin{aligned}
\left\{\underline{\widehat{b}}: \underline{\widehat{b}}^{\dagger} \widehat{\Lambda}:\right\} & =\frac{\Omega}{N}\left[-\underline{\underline{I}}\left(\underline{\underline{\sigma}}^{-1}-\underline{\underline{I}}\right) \underline{\underline{\sigma}} \frac{\mathrm{d} \widehat{\Lambda}^{(u)}}{\mathrm{d} \underline{\underline{\sigma}}} \underline{\underline{\sigma}}-\underline{\underline{I}}^{(u)}\right] \\
& =-\underline{\underline{\sigma}}\left(\frac{\mathrm{d} \widehat{\Lambda}}{\mathrm{d} \underline{\underline{\sigma}}}+\frac{\widehat{\Lambda}}{N} \frac{\mathrm{d} N}{\mathrm{~d} \underline{\underline{\sigma}}}\right) \underline{\underline{\sigma}}-\underline{\underline{I} \widehat{\Lambda}} \\
& =-\underline{\underline{\sigma}}\left(\frac{\mathrm{d} \widehat{\Lambda}}{\mathrm{d} \underline{\underline{\sigma}}}-\widehat{\Lambda} \underline{\underline{\sigma}}^{-1}\right) \underline{\underline{\sigma}}-\underline{\underline{I} \widehat{\Lambda}} \\
& =-\underline{\underline{\sigma}}-\underline{\underline{\sigma}} \frac{\mathrm{d} \hat{\Lambda}}{\mathrm{d} \underline{\underline{\sigma}}} \underline{\underline{\sigma}} .
\end{aligned}
$$

\section{B.7. Antinormal products}

Theorem 6. An antinormally ordered product of a pair of ladder operators and a Gaussian is equivalent to a first-order differential operator on the Gaussian, as follows:

$$
\{\underline{\widehat{b}} \underline{\underline{b}} \hat{\Lambda}\}=-\underline{\underline{\sigma}} \widehat{\underline{\Lambda}}-\underline{\underline{\sigma}} \frac{\partial \widehat{\Lambda}}{\partial \underline{\underline{\alpha}}} \underline{\underline{\sigma}} .
$$

Proof. The proof initially proceeds in the same manner as for products of mixed ordering. We first insert the coherent-state identity to convert the action of the operators into integrals over coherent-state projectors:

$$
\begin{aligned}
&\{\underline{\widehat{b}} \underline{\hat{b}} \hat{\Lambda}\}=\int \mathrm{d} \underline{\gamma} \mathrm{d} \underline{\mathrm{\beta}} \mathrm{d} \underline{\alpha} \mathrm{d} \underline{\epsilon}|\gamma\rangle\left\langle\gamma\left|\left\{\underline{\widehat{b} \widehat{b}^{\dagger}}|\boldsymbol{\beta}\rangle\langle\boldsymbol{\beta}|\widehat{\Lambda}| \boldsymbol{\alpha}\rangle\langle\boldsymbol{\alpha}|\right\}\right| \epsilon\right\rangle\langle\epsilon| \\
&=\frac{\Omega}{N} \int \mathrm{d} \underline{\gamma} \mathrm{d} \underline{\beta} \mathrm{d} \underline{\alpha} \mathrm{d} \underline{\epsilon}|\gamma\rangle\langle\epsilon| \exp \left[-\frac{1}{2}(\overline{\boldsymbol{\beta}}, \boldsymbol{\alpha}) \underline{\underline{\mu}}\left(\frac{\boldsymbol{\alpha}}{\overline{\boldsymbol{\beta}}}\right)\right] \\
& \times\left(\begin{array}{c}
\boldsymbol{\beta} \\
\overline{\boldsymbol{\alpha}}
\end{array}\right)(\overline{\boldsymbol{\alpha}}, \boldsymbol{\beta}) \exp \left[\bar{\gamma} \boldsymbol{\beta}+\overline{\boldsymbol{\alpha}} \boldsymbol{\epsilon}-\overline{\boldsymbol{\alpha}} \boldsymbol{\alpha}-\overline{\boldsymbol{\beta}} \boldsymbol{\beta}-\frac{1}{2} \bar{\gamma} \gamma-\frac{1}{2} \bar{\epsilon} \epsilon\right] .
\end{aligned}
$$


This time, however, we integrate by parts twice:

$$
\begin{aligned}
\exp [-(\overline{\boldsymbol{\beta}}, \boldsymbol{\alpha}) \underline{\tilde{\mu}}= & \left.\left(\begin{array}{c}
\boldsymbol{\alpha} \\
\boldsymbol{\beta}
\end{array}\right) / 2\right]\left(\begin{array}{c}
\boldsymbol{\beta} \\
\overline{\boldsymbol{\alpha}}
\end{array}\right)(\overline{\boldsymbol{\alpha}}, \boldsymbol{\beta}) \exp (-\overline{\boldsymbol{\alpha}} \boldsymbol{\alpha}-\overline{\boldsymbol{\beta}} \boldsymbol{\beta}) \\
= & \exp \left[-(\overline{\boldsymbol{\beta}}, \boldsymbol{\alpha}) \underline{\underline{\tilde{\mu}}}\left(\begin{array}{c}
\boldsymbol{\alpha} \\
\overline{\boldsymbol{\beta}}
\end{array}\right) / 2\right]\left(\begin{array}{c}
-\partial_{\overline{\boldsymbol{\beta}}} \\
\partial_{\boldsymbol{\alpha}}
\end{array}\right)\left(\partial_{\alpha},-\partial_{\overline{\boldsymbol{\beta}}}\right) \exp (-\overline{\boldsymbol{\alpha}} \boldsymbol{\alpha}-\overline{\boldsymbol{\beta}} \boldsymbol{\beta}) \\
\rightarrow & \exp (-\overline{\boldsymbol{\alpha}} \boldsymbol{\alpha}-\overline{\boldsymbol{\beta}} \boldsymbol{\beta})\left(\begin{array}{c}
-\partial_{\overline{\boldsymbol{\beta}}} \\
\partial_{\boldsymbol{\alpha}}
\end{array}\right)\left(\partial_{\alpha},-\partial_{\overline{\boldsymbol{\beta}}}\right) \exp \left[-\frac{1}{2}(\overline{\boldsymbol{\beta}}, \boldsymbol{\alpha}) \underline{\underline{\mu}}\left(\frac{\boldsymbol{\alpha}}{\overline{\boldsymbol{\beta}}}\right)\right] \\
= & \exp (-\overline{\boldsymbol{\alpha}} \boldsymbol{\alpha}-\overline{\boldsymbol{\beta}} \boldsymbol{\beta})\left[-\underline{\underline{I}}+\underline{\underline{I}} \underline{\underline{\mu}}\left(\frac{\boldsymbol{\alpha}}{\overline{\boldsymbol{\beta}}}\right)(\overline{\boldsymbol{\beta}}, \boldsymbol{\alpha})\right] \underline{\underline{\mu}} \underline{\underline{\underline{I}}} \\
& \times \exp \left[-\frac{1}{2}(\overline{\boldsymbol{\beta}}, \boldsymbol{\alpha}) \underline{\underline{\mu}}\left(\frac{\boldsymbol{\alpha}}{\overline{\boldsymbol{\beta}}}\right)\right]
\end{aligned}
$$

which is now in a form that we can again express as a derivative of the unnormalized Gaussian operator with respect to $\underline{\underline{\mu}}$ :

$$
\begin{aligned}
& \left\{\underline{\underline{b}} \underline{b}^{\dagger} \widehat{\Lambda}\right\}=\frac{-\Omega}{N} \int \mathrm{d} \underline{\gamma} \mathrm{d} \underline{\beta} \mathrm{d} \underline{\alpha} \mathrm{d} \underline{\epsilon}|\gamma\rangle\langle\epsilon| \exp \left[-\overline{\boldsymbol{\alpha}} \boldsymbol{\alpha}-\frac{1}{2} \overline{\boldsymbol{\beta}} \boldsymbol{\beta}-\bar{\gamma} \gamma-\frac{1}{2} \overline{\boldsymbol{\epsilon}} \boldsymbol{\epsilon}+\bar{\gamma} \boldsymbol{\beta}+\overline{\boldsymbol{\alpha}} \boldsymbol{\epsilon}\right] \\
& \times\left(\left[\underline{\underline{I}}-\underline{\underline{I}} \underline{\underline{\mu}} \frac{\mathrm{d}}{\underline{\mathrm{d}} \underline{\underline{\mu}}}\right] \exp \left[-\frac{1}{2}(\overline{\boldsymbol{\beta}}, \boldsymbol{\alpha}) \underline{\underline{\mu}}\left(\frac{\boldsymbol{\alpha}}{\overline{\boldsymbol{\beta}}}\right)\right]\right) \underline{\underline{\mu}} \underline{\underline{I}} \\
& =\frac{\Omega}{N}\left[-\underline{\underline{I}} \widehat{\Lambda}^{(u)}+\underline{\underline{I}} \underline{\underline{\mu}} \frac{\mathrm{d} \widehat{\Lambda}^{(u)}}{\mathrm{d} \underline{\underline{\mu}}}\right] \underline{\underline{\mu}} \underline{\underline{I}} .
\end{aligned}
$$

Finally, we change variables to $\underline{\underline{\sigma}}=[\underline{\underline{\mu}}+\underline{\underline{I}}]^{-1}$ and use the result for the derivative of the normalization:

$$
\begin{aligned}
\{\underline{\widehat{b} \underline{b}} \hat{\Lambda}:\} & =-\frac{\Omega}{N}\left[\underline{\underline{I}} \widehat{\Lambda}^{(u)}+\underline{\underline{\sigma}} \frac{\mathrm{d} \widehat{\Lambda}^{(u)}}{\mathrm{d} \underline{\underline{\sigma}}} \underline{\underline{\sigma}}\right] \underline{\underline{\sigma}}^{-1} \underline{\underline{\sigma}} \\
& =-\underline{\underline{\sigma}} \widehat{\Lambda}-\underline{\underline{\sigma}} \frac{\mathrm{d} \widehat{\Lambda}}{\mathrm{d} \underline{\underline{\sigma}}} \underline{\underline{\sigma}} .
\end{aligned}
$$

\section{References}

[1] Louisell W H 1973 Quantum Statistical Properties of Radiation (New York: Wiley)

[2] Smith A M and Gardiner C W 1988 Phys. Rev. A 384073

[3] Wigner E P 1932 Phys. Rev. 40749

[4] Husimi K 1940 Proc. Phys. Math. Soc. Japan 22264

[5] Glauber R J 1963 Phys. Rev. 1312766

Sudarshan E C G 1963 Phys. Rev. Lett. 10277

[6] Cahill K E and Glauber R J 1969 Phys. Rev. 1771882

[7] Chaturvedi S, Drummond P D and Walls D F 1977 J. Phys. A: Math. Gen. 10 L187-92

[8] Drummond P D and Gardiner C W 1980 J. Phys. A: Math. Gen. 132353

[9] Corney J F and Drummond P D 2003 Phys. Rev. A 68063822

[10] Bardeen J, Cooper L N and Schrieffer J R 1957 Phys. Rev. 1081175

[11] Gardiner C W 1983 Handbook of Stochastic Methods (Berlin: Springer)

[12] Corney J F and Drummond P D 2004 Phys. Rev. Lett. 93260401

[13] Assaad F F, Werner P, Corboz P, Gull E and Troyer M 2005 Preprint cond-mat/0509149

[14] Chaturvedi S and Drummond P D 1999 Eur. Phys. J. B 8251

[15] Corney J F and Drummond P D 2004 Preprint cond-mat/0411712 
[16] Rombouts S and Heyde K 2003 Phys. Status Solidi b 23799

[17] Martin J L 1959 Proc. R. Soc. A 251543

Ohnuki Y and Kashiwa T 1978 Prog. Theor. Phys. 60548

[18] Cahill K E and Glauber R J 1999 Phys. Rev. A 591538

[19] Berezin F A 1966 The Method of Second Quantization (New York: Academic)

Lehmann D 2005 Mathematical Methods of Many-Body Quantum Field Theory (London: Chapman and Hall/CRC Press)

[20] Blaizot J-P and Ripka G 1986 Quantum Theory of Finite Systems (Cambridge, MA: MIT Press)

Negele J W and Orland H 1988 Quantum Many-Particle Systems (Cambridge, MA: Perseus Publishing)

[21] Plimak L I, Collett M J and Olsen M K 2001 Phys. Rev. A 64063409

[22] Drummond P D 1983 Phys. Rev. Lett. 501407

[23] Deuar P and Drummond P D 2002 Phys. Rev. A 66033812

[24] Deuar P and Drummond P D 2001 Comput. Phys. Commun. 142442 